\title{
Quantum phase transition as an interplay of Kitaev and Ising interactions
}

\author{
A. Langari, ${ }^{1,2,3}$ A. Mohammad-Aghaei, ${ }^{1}$ and R. Haghshenas ${ }^{1}$ \\ ${ }^{1}$ Department of Physics, Sharif University of Technology, P.O.Box 11155-9161, Tehran, Iran \\ ${ }^{2}$ Center of excellence in Complex Systems and Condensed Matter (CSCM), \\ Sharif University of Technology, Tehran 1458889694, Iran \\ ${ }^{3}$ Max-Planck-Institut für Physik komplexer Systeme, 01187 Dresden, German) *
}

(Dated: October 9, 2014)

\begin{abstract}
We study the interplay between the Kitaev and Ising interactions on both ladder and two dimensional lattices. We show that the ground state of the Kitaev ladder is a symmetry-protected topological (SPT) phase, which is protected by a $\mathbb{Z}_{2} \times \mathbb{Z}_{2}$ symmetry. It is confirmed by the degeneracy of the entanglement spectrum and non-trivial phase factors (inequivalent projective representations of the symmetries), which are obtained within infinite matrix-product representation of numerical density matrix renormalization group. We derive the effective theory to describe the topological phase transition on both ladder and two-dimensional lattices, which is given by the transverse field Ising model with/without next-nearest neighbor coupling based on the primary Ising configurations. The ladder has three phases, namely, the Kitaev SPT, symmetry broken ferro/antiferromagnetic order and classical spin-liquid. The non-zero quantum critical point and its corresponding central charge are provided by the effective theory, which are in full agreement with the numerical results, i.e., the divergence of entanglement entropy at the critical point, change of the entanglement spectrum degeneracy and a drop in the ground-state fidelity. The central charge of the critical points are either $\mathrm{c}=1$ or $\mathrm{c}=2$, with the magnetization and correlation exponents being $1 / 4$ and $1 / 2$, respectively. The transition from the classical spin-liquid phase of the frustrated Ising ladder to the Kitaev SPT phase is mediated by a floating phase, which shows strong finite entanglement scaling. In the absence of frustration, the $2 \mathrm{D}$ lattice shows a topological phase transition from the $\mathbb{Z}_{2}$ spin-liquid state to the long-range ordered Ising phase at finite ratio of couplings, while in the presence of frustration, an order-by-disorder transition is induced by the Kitaev term. The 2D classical spin-liquid phase is unstable against the addition of Kitaev term toward an ordered phase before the transition to the $\mathbb{Z}_{2}$ spin-liquid state.

PACS numbers: 05.30.Rt, 75.10.Jm, 03.67.-a
\end{abstract}

\section{INTRODUCTION}

Topologically ordered quantum many-body systems have received a great deal of interest due to rich insights emerged from their nature, namely, lack of any local order parameter to characterize them ${ }^{1.2}$, i.e. failure of symmetry breaking paradigm, exhibiting long-range entanglement ${ }^{3}$, robustness against local perturbations ${ }^{4.5}$, non-trivial anyon statistics ${ }^{6.7}$ and so on. Topological quantum codes including color codes $\underline{8}-11$ have a universal feature characterized by topological entanglement entropy $\frac{12,13}{2}$ manifesting their topological nature. Emergent fermions and anyons ${ }^{14}-16$ are typical quasi-particle excitations above a topological ground-state that influence the finite temperature properties with non-trivial limiting features $\frac{17}{}$ and bound states $\frac{18}{}$. The stability of topological ordered state against thermal, external magnetic field ${ }^{19}$ and other interactions ${ }^{20}$ (like Ising ${ }^{21}$ ) is an interesting issue, which could lead to the phase transition from a topological state. For instance, an in-plane magnetic field on the toric code leads to both first- and second-order quantum phase transition ${ }^{22}$, while a perpendicular magnetic field gives a first-order phase transition at the self-dual point of the effective quantum compass model ${ }^{23}$. The $2 \mathrm{D}$ color code shows similar behavior in the presence of a magnetic field ${ }^{24}$ and Ising interactions ${ }^{25}$. The nature of such phase transition and its corresponding quantum critical properties are debating issues inherited from the topological properties of the model.
Recently, many efforts, inspired by the concepts of quantum information theory, have been made to provide a comprehensive understanding of topological order ${ }^{26-28}$. So far, it is believed there exists three different kinds of topological orders, namely: symmetry protected topological (SPT) order, long range entangled states with topological order and symmetry enriched topological order. The picture in $1 \mathrm{D}$ is complete and the symmetry-fractionalization mechanism competently characterize the SPT phases. In higher dimensions, it is believed that symmetry fractionalization, symmetry breaking and long-range entanglement mechanisms are capable of characterizing the aforementioned orders. However, to get a complete understanding, further studies are currently active and demanding.

Characterization of topological order relies on appropriate non-local order parameters. In $1 \mathrm{D}$, entanglement spectrum distinguishes SPT orders from trivial ones ${ }^{26}$, while non-local order parameters based on inequivalent projective representations $\mathrm{s}^{29}$ identifies different SPT orders ${ }^{30}$. In 2D, even so, there is not a unique and faithful tool to classify them but topological entanglement entropy ${ }^{13}$ is assumed as the most common tool to characterize intrinsic topological phases. The case of symmetry enriched topological orders is more complicated as both orders, i.e. SPT and intrinsic topological orders, simultaneously exist-some proposed non-local measures might hopefully identify them ${ }^{31,32}$. Understanding a quantum phase transition (QPT) from a topological phase to 
a trivial phase requires less effort than the classification of phases, since local-order parameters according to symmetry breaking mechanism can identify the quantum phase transition. Novel quantum phase transitions, which rarely have been studied, happen when there are two distinct topological phases.

In this article, we consider the Kitaev toric code model accompanied by different Ising interactions, namely, rhombicIsing (RI), leg-Ising (LI) and rhombic-leg-Ising (RLI) interactions, on ladder and two-dimensional square geometries. The non-frustrated RI case of ladder and 2D square lattice have been studied recently in Ref. 21. Here, we consider all possible Ising interactions, which include the frustrated models for both ladder and 2D lattices. The Kitaev toric code ${ }^{5}$ is a well-known model showing topological order, while Ising model with respect to the geometry of lattice and the type of interactions can show up symmetry broken phases and topological spin-liquid phases ${ }^{33}$. The later is due to frustration of anti-ferromagnetic (AF) interactions of Ising model (AF RLI interactions), which leads to a rich phase diagram ${ }^{34} \underline{42}$.

We show that the ground state of the Kitaev ladder is an SPT phase by introducing the responsible $\mathbb{Z}_{2} \times \mathbb{Z}_{2}$ symmetries, which is confirmed by numerical results on the corresponding non-trivial phase factor ${ }^{30}$ - see appendix $B$, To investigate the competition between the Kitaev SPT phase and an Ising phase we employ two general approaches: an effective theory, which comes from an exact map of the original model to an effective one, and the infinite system densitymatrix-renormalization-group (iDMRG) algorithm ${ }^{43-45}$ based on infinite matrix product state (iMPS) representation. On the ladder geometry, the Kitaev plus Ising interactions are mapped to decoupled chains of nearest-neighbor $(\mathrm{NN})$ or next-nearestneighbor (NNN) transverse field Ising (TFI) model. The effective theory and numerical iDMRG computations show a quantum phase transition at finite non-zero coupling from the Kitaev SPT phase to the broken symmetry antiferro/ferromagnetic phase except in the case of AF RLI interactions. The existence of quantum critical point (QCP) and its location is proved by numerical simulation that leads to the divergence of the entanglement entropy, change in the degeneracy of the entanglement spectrum, drop in the ground-state fidelity, change of phase factor, and non-zero magnetic order parameter. We have also computed the corresponding central charges, which is in agreement with the proposed effective theory, namely, $c=2$ for Kitaev-LI, $c=1$ for both Kitaev-RI and Kitaev-RLI QCPs. The critical exponents of magnetization and correlation function are $\beta=1 / 4$ and $\eta=1 / 2$, correspondingly for all types of non-frustrated ladders. Concerning the QCP, our result for Kitaev-RI interactions is in contrast to Ref. 21, which concludes zero Ising coupling strength, while we observe a finite non-zero Ising coupling $\left(J_{z}=J_{v}\right.$, see Fig. 5). Moreover, the whole study for the LI and RLI cases are new investigations of this manuscript that includes the frustrated case. The case of AF RLI coupling makes a competition between the Kitaev SPT phase and a classical spin-liquid one, which can be explained in terms of the frustrated NNN TFI effective theory. Our numerical results for AF
RLI case are in favor of the existence of a floating phase which has algebraic decaying correlations-between the classical spin-liquid and Kitaev SPT phases. This is in agreement with the phase diagram proposed for the (effective) frustrated NNN TFI chain in Refs. 40 and 41.

For 2D square lattice, we follow the same strategy and map the original model to an effective theory, which is given by (NN or NNN) 2D TFI model in terms of (effective) quasispins. The effective theory is defined on the bi-partite square lattice of quasi-spins, where the two sub-lattices are decoupled. For all types of Ising interactions except the case of AF Ising interactions on all bonds, we get a QPT at finite non-zero couplings from the intrinsic topological $\left(\mathbb{Z}_{2}\right.$ spin-liquid) phase to a long-range ordered of ferro or antiferromagnetic (Néel) type. At the extreme limit of AF Ising coupling on all bonds, the presence of frustration leads to extensive degenerate configurations, which would be destabilized by order-by-disorder transition $\stackrel{46}{-4} \underline{-48}$ due to fluctuations induced by Kitaev term. At higher values of Kitaev term a transition to the topological $\mathbb{Z}_{2}$ spin-liquid phase occurs.

The remainder of this paper is organized as follows. In Sec. II we first briefly review the Kitaev toric code on ladder and introduce the $\mathbb{Z}_{2} \times \mathbb{Z}_{2}$ symmetry, which protects the degeneracy of the entanglement spectrum. We then define different types of Ising interactions in Sec. III and derive the effective theory for the Kitaev-Ising interplay. We present our numerical results in the same section. We consider the $2 \mathrm{D}$ version of the interplay between Kitaev and Ising interaction in Sec. IV] where an effective theory is introduced for all types of Ising couplings. Finally in Sec. $\mathrm{V}$ we end up with a summary and discussion. The article is accompanied by two appendices, which describe the underlying numerical iDMRG (iMPS) approach.

\section{THE SPT PHASE OF KITAEV LADDER}

The Kitaev ladder is defined on the ladder geometry as shown in Fig. 1, where the spins sit on the bonds of the twoleg ladder. The Kitaev Hamiltonian $\left(\mathcal{H}_{\mathcal{K}}\right)$ is composed of two terms, vertex $\left(A_{v}\right)$ and plaquette $\left(B_{p}\right)$ interactions

$$
\begin{array}{r}
\mathcal{H}_{\mathcal{K}}=-J_{v} \sum_{\perp, \top} A_{v}-J_{p} \sum_{\square} B_{p}, \\
A_{v} \equiv \prod_{i} \sigma_{i}^{x}, \quad i \in \perp \text { or } \top ; \quad J_{v}>0, \\
B_{p} \equiv \prod_{j} \sigma_{j}^{z}, \quad j \in \square ; \quad J_{p}>0,
\end{array}
$$

where $\sigma_{j}^{\alpha}$ is the $\alpha$-component Pauli matrix at position $j$. The model is exactly solvable $\underline{49}$, which has two-fold topologically degenerate ground states. Let $|\Omega\rangle \equiv \otimes_{i}|+\rangle_{i}$, where $|+\rangle_{i}$ is the eigenstate of $\sigma_{i}^{x}$, a ground state of Kitaev ladder is given by

$$
\left|\psi_{K}\right\rangle=\frac{1}{2^{N}} \prod_{p}\left(1+B_{p}\right)|\Omega\rangle,
$$




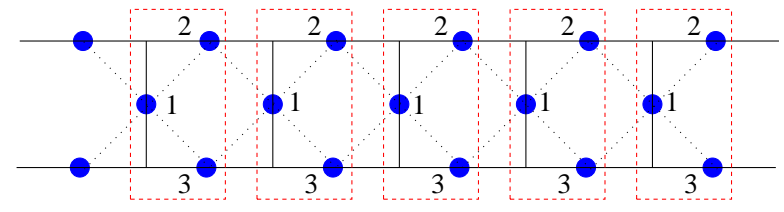

FIG. 1. (color online) Two-leg Kitaev ladder, where the filled-blue circles show the position of real spins. The triangles show the vertex term and rhombuses represent the plaquette term in the Hamiltonian. The dashed-red rectangle shows the unit cell of the model.

where $N$ is the total number of rungs on the ladder. And $\left|\psi_{K}^{\prime}\right\rangle=W_{z}\left|\psi_{K}\right\rangle$ is the other ground state in which $W_{z}=$ $\prod_{\ell} \sigma_{\ell}^{z}$, where $\ell$ runs only on one of the legs of ladder. The ground state is understood as an equally weighted superposition of the states, which are obtained by the operation of any homologically trivial loop of $\sigma^{z}$ operators on $|\Omega\rangle$. The excited states can be constructed by the operation of open strings of $\sigma^{z}$ operators on $|\Omega\rangle$. A complete characterization of the spectrum shows that the excited states are at least two-fold degenerate, which could be more except the highest energy level that has only a double degeneracy $\stackrel{49}{ }$. Moreover, the ground state entropy of the model is equal to $\ln (2)$.

The Kitaev ladder $\left(\mathcal{H}_{\mathcal{K}}\right)$ has (i) two-fold degenerate ground state which can not be distinguished by a local-order parameter of the Landau-Ginzburg symmetry breaking paradigm, (ii) a finite-energy gap between the ground state and the first excited one and (iii) anyonic excitations of integer magnetic and electric charges with Abelian statistics ${ }^{49}$. Although the quasione dimensional Kitaev ladder does not bear topological characters like Wilson loops and topological entanglement entropy its ground state is classified to be an SPT phase. We will show explicitly that the ground state of Kitaev ladder is protected by a $\mathbb{Z}_{2} \times \mathbb{Z}_{2}$ symmetry. For each unit cell of the two-leg ladder, Fig. 11 the following operator is defined,

$$
\Sigma^{a b c}(j)=\sigma_{1}^{a}(j) \sigma_{2}^{b}(j) \sigma_{3}^{c}(j), \quad a, b, c=I, x, y, z,
$$

where $\sigma_{i}^{I}(j)$ is the identity operator at position $i$ of unit cell $j$. It is straight forward to show that $\mathcal{H}_{\mathcal{K}}$ is invariant under the operation of the following two operators,

$$
\mathcal{X}=\prod_{j} \Sigma^{x x x}(j) \text { and } \mathcal{Z}=\prod_{j} \Sigma^{I z z}(j),
$$

where $j$ runs over all unit cells. Moreover, $\left[\mathcal{X}, B_{p}\right]=0$, which states that the ground state of Kitaev-ladder is invariant under $\mathcal{X}$. Similarly, it can be shown that the ground state of Kitaev-ladder is invariant under $\mathcal{Z}$, since the product of $\mathcal{Z}$ by $\prod_{p}\left(1+B_{p}\right)$ is equivalent to the operation of $\prod_{p}\left(1+B_{p}\right)$. Hence, the Kitaev-ladder ground state is invariant under the mutual symmetry operation $\mathcal{X} \times \mathcal{Z}$, which defines the mentioned $\mathbb{Z}_{2} \times \mathbb{Z}_{2}$ symmetry. The local symmetry operation $\Sigma^{I z z}, \Sigma^{x x x}$, are two members of the group $G=\left\{\Sigma^{I z z}, \Sigma^{x x x},-\Sigma^{x y y}, \Sigma^{I I}\right\}$. According to $\operatorname{Ref} 30$, we exploit this property and define an order parameter $\mathcal{O}$, which can serve to detect, which projective representation holds for the ground state in terms of its iMPS representation. The order parameter $\mathcal{O}$ is defined by

$$
\mathcal{O}=\frac{1}{\chi} \operatorname{Tr}\left(U_{g} U_{g^{\prime}} U_{g}^{\dagger} U_{g^{\prime}}^{\dagger}\right), \quad g \in G
$$

where $U_{g}$ comes from the transformation of iMPS representation of the ground state $\left(\Gamma_{j}\right)$ under the symmetry $\mathcal{X} \times \mathcal{Z}$, i.e. $\Gamma_{j} \rightarrow U_{g}^{\dagger} \Gamma_{j} U_{g}$, where $g \in G$ and $\chi$ is the dimension of matrices in iMPS (for details see appendix-B). If the ground state respects the symmetry, $\mathcal{O}=1$ for a trivial phase and $\mathcal{O}=-1$ for an SPT phase. Otherwise, when the ground state is not invariant under symmetry we get $\mathcal{O}=0$, which shows the presence of symmetry breaking phenomenon. $\mathcal{O}$ is called "phase factor order parameter". We find numerically that the Kitaev phase reveals $\mathcal{O}=-1$, (see Fig. 9) which justifies that it is being protected by $\mathcal{X} \times \mathcal{Z}$ symmetries. Moreover, the entanglement spectrum is degenerate in the Kitaev phase (see Fig. (4), which confirms that the ground state of Kitaev-ladder is an SPT phase.

\section{KITAEV-ISING LADDER}

The Ising term, which is composed of two-body interactions, competes with the SPT character of the pure Kitaev ground state on the ladder. The Ising interaction $\sigma_{i}^{z} \sigma_{j}^{z}$, (which is defined on the nearest neighbor spins of ladder) does not commute with the vertex terms $\left(A_{v}\right)$ of $\mathcal{H}_{\mathcal{K}}$ that establishes a competitions between a symmetry-protected topological and a classical state. The classical state, which is a result of strong Ising interaction could be realized in different forms according to the pattern of Ising interactions. We classify three types of Ising interactions on the two-legs ladder in Fig. 1 (A) Rhombic-Ising interactions, where the Ising terms are defined only between the nearest neighbor spins sitting on each rhombus. The corresponding Hamiltonian $\left(\mathcal{H}_{\mathcal{R}}\right)$ is defined in Eq. (10). (B) Leg-Ising interactions, which is defined between nearest neighbor spins on the legs of ladder and given by $\mathcal{H}_{\mathcal{L}}$ in Eq16 (C) Rhombic-leg Ising interactions that is composed of nearest neighbor interaction between any pair of spins on the two-legs ladder, which is being represented by the sum of two previous cases, i.e. $\mathcal{H}_{\mathcal{R} \mathcal{L}}=\mathcal{H}_{\mathcal{R}}+\mathcal{H}_{\mathcal{L}}$. We consider both ferromagnetic $\left(J_{z}>0\right)$ and anti-ferromagnetic $\left(J_{z}<0\right)$ coupling for the Ising terms. The latter leads to a rich structure of the ground state phase diagram as a result of frustration originated from the anti-ferromagentic interactions on the bonds of triangles (see Fig. 2).

To investigate the competition between Kitaev and Ising interactions, we introduce a transformation that gives the effective theory, which illustrates the quantum phase transition of Kitaev model in the presence of Ising interactions. The Hamiltonian is composed of three types of terms, i.e. the vertex $\left(A_{v}\right)$, plaquette $\left(B_{p}\right)$ and Ising $\left(\sigma_{i}^{z} \sigma_{j}^{z}\right)$ terms. The plaquette term commutes with both vertex and Ising ones, $\left[B_{p}, A_{v}\right]=0,\left[B_{p}, \sigma_{i}^{z} \sigma_{j}^{z}\right]=0$, and consequently does not play any role in the competition for quantum phase transition. 


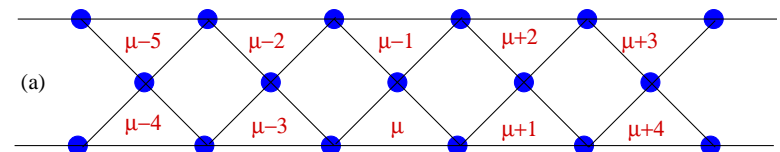

(b)
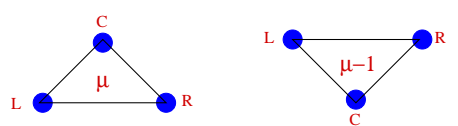

FIG. 2. (color online) (a) The Kitaev ladder, where each vertex operator (triangle) is denoted as an effective spin $\left(\tau_{\mu}^{z}\right)$ labeled by $\mu$. (b) The original spins are relabeled by the triangle index $(\mu)$ and its position (L, R, C) on it.

However, the plaquette term adds a constant term to the underlying Hamiltonian, which is being fixed to its minimum value for the ground state properties, i.e. $B_{p}=+1$.

The building block of the effective theory is a triangle that is denoted by a vertex operator. To visualize this picture, the ladder is labeled by its triangles corresponding to each vertex operator in Fig. 2. (a). In this representation, the spin of the original lattice carries two indices, the label of triangle $(\mu)$ and a label, which sticks to right (R), left (L) or center (C) of a triangle, as can be seen in Fig. 2.(b). A vertex operator is then given by

$$
A_{\mu}=\sigma_{\mu, L}^{x} \sigma_{\mu, R}^{x} \sigma_{\mu, C}^{x} .
$$

We consider the $\mathrm{x}$-representation as the basis of our study. In this representation, a vertex operator $\left(A_{\mu}\right)$ has two values either +1 or -1 , which is denoted by the associated quasi-spin $\left(\tau_{\mu}^{z}\right)$, i.e.

$$
A_{\mu} \longrightarrow \tau_{\mu}^{z}
$$

It concludes that the effect of Kitaev Hamiltonian on the quasi-spin representation is like a magnetic filed,

$$
J_{v} \sum_{v} A_{v} \longrightarrow J_{v} \sum_{\mu} \tau_{\mu}^{z}
$$

The effect of a single $\sigma_{\mu, L}^{z}$ on a quasi-spin (a triangle) is to flip its state, which is denoted by $\tau_{\mu}^{x}$ in the quasi-spin representation,

$$
\sigma_{\mu, m}^{z} \longrightarrow \tau_{\mu}^{x}, \quad m=L, R, C .
$$

Depending on the geometry, where Ising interactions reside, we get three different effective models leading to distinct critical points and universal behaviors.

\section{A. Rhombic-Ising interactions}

The Rhombic-Ising terms are those two-body spin interactions, which act only along the edges of Rhombic shapes in

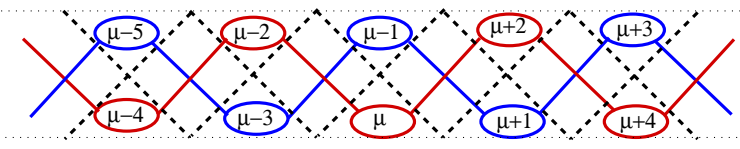

FIG. 3. (color online) The effective model, which represents the Kitaev ladder in the presence of rhombic-Ising interactions that is composed of two decoupled Ising chains in a transverse field. An oval represents a quasi-spin $(\tau)$.

Fig. 2. (a). According to the notation presented in Fig. 2.-(b) the Ising Hamiltonian is given by

$$
\mathcal{H}_{\mathcal{R}}=-J_{z} \sum_{\mu}\left(\sigma_{\mu, C}^{z} \sigma_{\mu, R}^{z}+\sigma_{\mu, C}^{z} \sigma_{\mu, L}^{z}\right)
$$

It is important to note that the Rhombic-Ising terms do not change the state of a quasi-spin, which shares an edge with the rhombus. For instance, $\sigma_{\mu, C}^{z} \sigma_{\mu, R}^{z}$ flips two times the state of the triangle denoted by $\mu$, which leads to its original state (see Fig. 2-(b)). However, the state of a triangle that only shares a single spin at its corner, is flipped. In other words, the operation of $\sigma_{\mu, C}^{z} \sigma_{\mu, R}^{z}$ flips the state of quasi-spins (triangles) denoted by $\mu-1$ and $\mu+1$. Therefore, each Ising term, like $\sigma_{\mu, C}^{z} \sigma_{\mu, R}^{z}$ is represented by the product of two x-component quasi-spin acting as $\tau_{\mu-1}^{x} \tau_{\mu+1}^{x}$. The Ising interactions on the edges of a rhombus create effective interactions between the quasi-spins corresponding to the adjacent edges. It leads to the effective interaction, $2 J_{z} \tau_{\mu-1}^{x} \tau_{\mu+1}^{x}$, between two odd or two even quasi-spins, independently (see Fig. 3). Thus, the effective Hamiltonian $\left(\mathcal{H}_{e f f}^{\mathcal{R}}\right)$, which describes the Kitaev Hamiltonian in the presence of Rhombic-Ising interactions, $\mathcal{H}_{\mathcal{K}}+\mathcal{H}_{\mathcal{R}}$, is given by two decoupled chain of transverse field Ising (TFI) model,

$$
\mathcal{H}_{\text {eff }}^{\mathcal{K R}}=-2 J_{z} \sum_{\mu}^{\prime} \tau_{\mu}^{x} \tau_{\mu+2}^{x}-J_{v} \sum_{\mu}^{\prime} \tau_{\mu}^{z}, \quad \mu=\text { odd or even }
$$

where $\sum_{\mu}^{\prime}$ emphasizes the odd and even quasi-spins are decoupled. Accordingly, a quantum phase transition takes place exactly at $2 J_{z}=J_{v}$, which is known from the exact solution of spin-1/2 TFI chain. Our result is in contrast to $J_{z}^{c}=0$ presented in Ref 21. In Ref 21 the Kitaev ladder with RhombicIsing interaction is mapped to a spin-1/2 XY chain .using a non-local transformation. Their mapping and the effective XY model are correct; however, the conclusion of zero Ising critical coupling $\left(J_{z}^{c}=0\right)$ overlooks the true QCP. The exactly solvable spin-1/2 XY chain ${ }^{50}$ is defined by the Hamiltonian $H_{X Y}=J_{x} \sum_{i}\left(s_{i}^{x} s_{i+1}^{x}+\gamma s_{i}^{y} s_{i+1}^{y}\right)$, where $\gamma \equiv J_{y} / J_{x}$. For any non-zero value of $\gamma$ the XY chain is gapped except $\gamma=1$, where the gap vanishes as $|\gamma-1|$ at momentum $q=\pi / 2$. The elementary excitations at the (gapless) critical point are spinons 51 . Moreover, the 2 nd derivative of ground state energy $\left(E_{0}\right)$ diverges as

$$
\frac{d^{2} E_{0}}{d \gamma^{2}} \sim|\gamma-1|^{-3} \quad \text { at } \quad q=\frac{\pi}{2}
$$




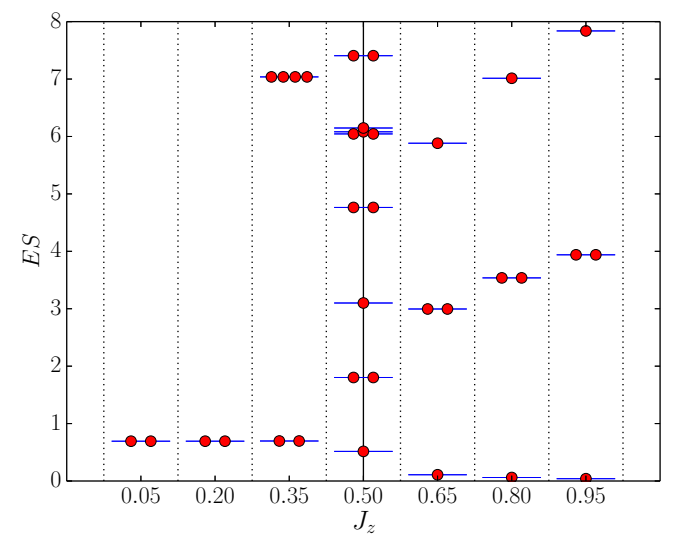

FIG. 4. (color online) Entanglement spectrum (ES) versus $J_{z}$ for the ground state of $\mathcal{H}_{\mathcal{K}}+\mathcal{H}_{\mathcal{R}}$. The lowest level is doubly degenerate for $0 \leq J_{z}<0.5$, which is a signature of SPT character, while it is non-degenerate for $J_{z}>0.5$ in the (trivial) ferromagnetic product state. At the quantum critical point $J_{z}=0.5$, the spectrum becomes dispersed over the entire range of eigenvalues, which is a signature of the critical point.

which justifies the quantum phase transition at the isotropic point, $\gamma=1$, that corresponds to our result $J_{z}^{c}=J_{v} / 2$.

To gain more insights on the structure of phases and the nature of quantum phase transition, we obtain, by numerical calculations, the ground state of Kitaev ladder in the presence of rhombic-Ising terms using an implementation of the iDMRG algorithm (see appendix A). The code is based on iMPS representation, where $\chi$ denotes the dimension of matrices in this formalism. The entanglement spectrum of the ground state is defined in terms of the eigenvalues of the reduced density matrix. Let $\rho$ be the ground-state reduced density matrix, which is obtained by tracing over half of the ladder from the middle to either the left or right end of ladder,

$$
\rho=\operatorname{tr}_{L / 2}\left(\left|\psi_{0}\right\rangle\left\langle\psi_{0}\right|\right),
$$

where $\left|\psi_{0}\right\rangle$ is the ground state of Kitaev with Ising interactions. Let $\lambda_{i}$ be the eigenvalues of $\rho$, the entanglement spectrum (ES) is defined by $\varepsilon_{i}=-\ln \left(\lambda_{i}\right)$. We have plotted the entanglement spectrum versus $J_{z}$ of Kitaev ladder in the presence of rhombic-Ising interactions in Fig. 4] which exhibits a change of degeneracy at $J_{z}=0.5$. We set $J_{v}=1$ as the scale of energy in all plots and results unless it appears explicitly. The spectrum is doubly degenerate for $0 \leq J_{z}<0.5$, which is a clear signature for the SPT character of Kitaev phase, while it is non-degenerate for $J_{z}>0.5$ in the (trivial) ferromagnetic product state. The change of degeneracy of the entanglement spectrum at $J_{z}=0.5$ is an indication of quantum phase transition, which is accompanied by a qualitative change in the ground state.

The von Neumann (entanglement) entropy $\left(S_{E}\right)$ is defined

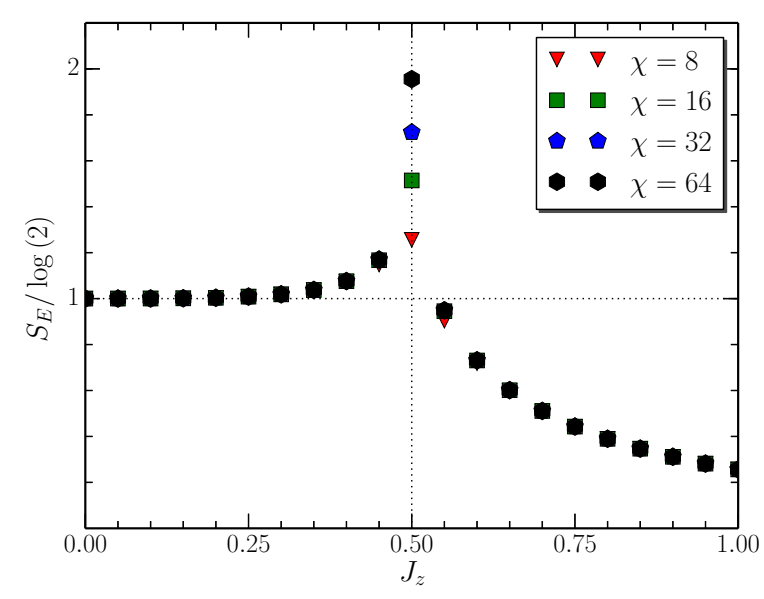

FIG. 5. (color online) von Neumann entropy $\left(S_{E}\right)$ versus $J_{z}$ for Kitaev plus rhombic-Ising interactions. The divergent behavior of $S_{E}$ at $J_{z}=0.5$ is a clear signature of quantum phase transition. $S_{E}$ reaches $\ln (2)$ asymptotically for the pure Kitaev ladder $\left(J_{z}=0\right)$.

in terms of the eigenvalues of $\rho$,

$$
S_{E}=-\sum_{i} \lambda_{i} \ln \left(\lambda_{i}\right)
$$

We have plotted $S_{E}$ versus $J_{z}$ in Fig. 5 for different $\chi=$ $8,16,32,64$. The entropy shows a divergent behavior only at $J_{z}=0.5$, which justifies the quantum phase transition. As shown in Fig. 5. $S_{E}$ asymptotically reaches the value of $\ln (2)$ for the pure Kitaev ladder $\left(J_{z}=0\right)$, which is the signature of its SPT character (the double degeneracy of ES), while it vanishes in the extreme Ising limit $\left(J_{z} \rightarrow \infty\right)$ representing a product state of up (or down) spins in a ferromagnetic state.

The ground-state fidelity $(\mathrm{F})$ is a specific measure to investigate a quantum phase transition without an ad-hoc assumption on the structure of ordering on any side of the transition point. The ground-state fidelity is defined by

$$
F\left(J_{z}, J_{z}+\delta J_{z}\right)=\left\langle\psi_{0}\left(J_{z}\right) \mid \psi_{0}\left(J_{z}+\delta J_{z}\right)\right\rangle,
$$

where $\delta J_{z}$ is a very small amount of change in the coupling constant, which gives rise to quantum phase transition. The ground-state fidelity is plotted in Fig. 6 versus $J_{z}$ for two different values of $\chi=16,64$ and $\delta J_{z}=0.01$. An obvious drop at $J_{z}=0.5$ confirms our observation of the quantum phase transition at $J_{z}=0.5$.

We have also computed the ordinary magnetic order parameter on both sides of the quantum critical point. We have plotted in Fig. 7 the magnetic order parameters in $x$ and $\mathrm{z}$ directions, $\left\langle\sigma_{x}\right\rangle$ and $\left\langle\sigma_{z}\right\rangle$, respectively. $\left\langle\sigma_{x}\right\rangle$ is always zero for the whole range of $J_{z}$, which shows no magnetic order in x-direction. However, $\left\langle\sigma_{z}\right\rangle$ becomes non-zero at $J_{z}=0.5$ indicating the magnetic order of the ferromagnetic state. Approaching the quantum critical point from the 


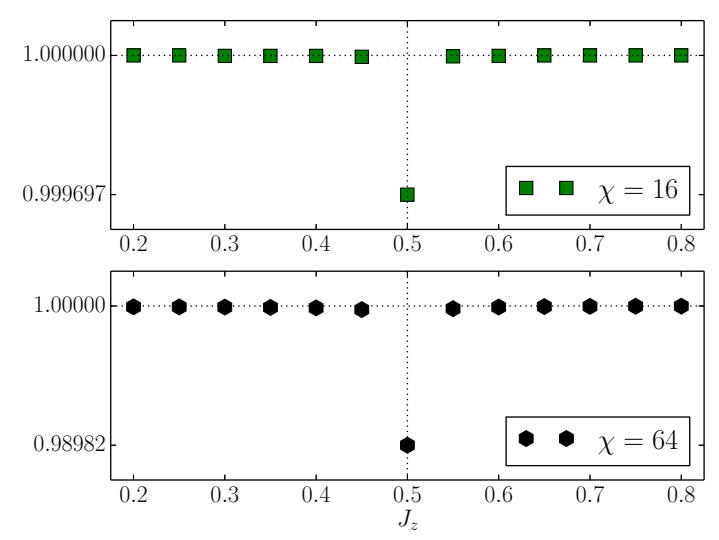

FIG. 6. (color online) Ground-state fidelity versus $J_{z}$ for Kitaev-RI interactions. A clear drop at $J_{z}=0.5$ justifies the quantum phase transition.

ferromagnetic phase $\left(J_{z}>0.5\right)$ shows $\left\langle\sigma_{z}\right\rangle$ to vanish like $(J-0.5)^{(0.24 \pm 0.01)}$ manifesting a second order phase transition with exponent $\beta=0.24 \pm 0.01$ (the inset of Fig. 7), in agreement with the effective theory described in Eq11. The effective theory, for Kitaev ladder with rhombic-Ising interactions, is expressed in terms of two decoupled TFI chains, which predicts the central charge for the corresponding QPT at $J_{z}=0.5$ is being twice the central charge of the TFI chain, i.e. $c=2 \times 0.5=1$. Similar argument shows that the magnetization exponent, which comes out of the effective theory, is $\beta=1 / 4$ (which is discussed in Sec $\mathbb{V}$ ). We have numerically calculated the central charge at the critical point $J_{z}=0.5$, which leads to $c=1.01 \pm 0.01$ as shown in Fig. 8f-(a). The central charge is calculated within finite-entanglement scaling introduced in $\operatorname{Ref} 52$. In this approach, the scaling of $S_{E}$ with the correlation length $(\xi)$ would give a fair approximation of the central charge, (see appendix A). The numerical result confirms that the effective theory truly captures the critical properties of the original model.

We have plotted the phase factor order parameter $\mathcal{O}$ versus $J_{z}$ in Fig. 9. It shows that for small values of $J_{z}<J_{z}^{c}$, the model is in the SPT phase of Kitaev ladder, which is justified by $\mathcal{O}=-1$. More specifically, Fig. 9 -(a) shows that for $J_{z}<0.5$ the model represents an SPT phase, while it shows a symmetry broken trivial phase $(\mathcal{O}=0)$ for $J_{z}>0.5$ via a quantum phase transition. The symmetry broken phase does not respect the symmetry, which gives the largest eigenvalue of the transfer matrix to be less than 1 , leading to $\mathcal{O}=0$.

Almost the whole discussion of the ferromagnetic rhombicIsing interaction is also valid for antiferromagnetic Ising interaction. In other words, we can simply consider a mirror image of all Figs. 4, 5, 6, 7 and 9 with respect to $J_{z}=0$ to get the antiferromagnetic regime. A better understanding can be achieved by considering a $\pi$ rotation around $\mathrm{x}$-axis for the spins sitting on the rungs of ladder and $J_{z} \rightarrow-J_{z}$, which

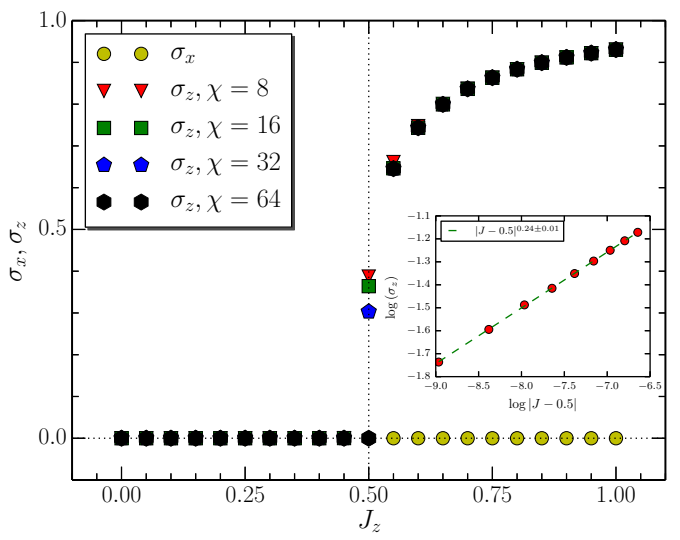

FIG. 7. (color online) Magnetic order parameters versus $J_{z}$ for Kitaev plus RI interactions. The ferromagnetic order parameter, $\left\langle\sigma_{z}\right\rangle$ becomes nonzero for $J_{z} \geq 0.5$ justifying the quantum phase transition to the symmetry broken state. The inset shows the scaling of $\left\langle\sigma_{z}\right\rangle \sim(J-0.5)^{(0.24 \pm 0.01)}$, where horizontal axis is in log-scale, close to the critical point.

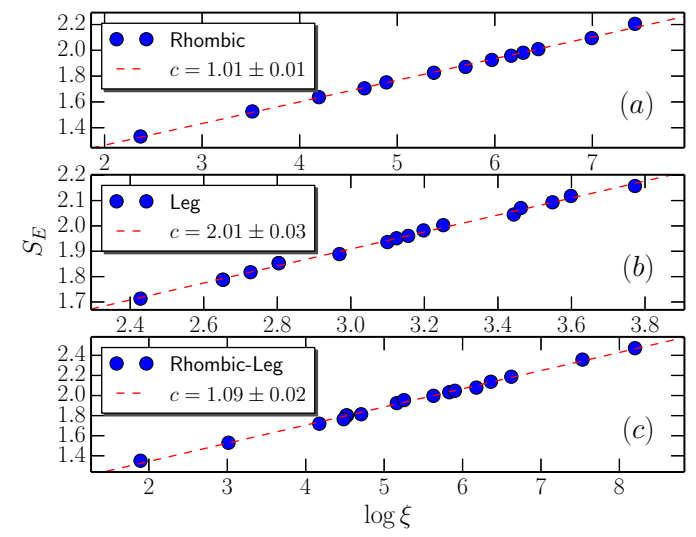

FIG. 8. (color online) $S_{E}$ versus $\log (\xi)$, which gives the central charge of the Kitaev ladder in addition to (a) RI, (b) LI and (c) RLI interactions according to the finite entanglement scaling . (see appendix $\mathrm{A}$ and Ref. 52 and 53 )

leaves the whole Hamiltonian invariant.

\section{B. Leg-Ising interactions}

The Ising terms may be considered only between the spins on the legs of ladder, without any inter-leg interaction. Therefore, the Ising interactions would be between spins labeled by $\mathrm{L}$ and $\mathrm{R}$, namely: $\sigma_{\mu, R}^{z} \sigma_{\mu, L}^{z}$, which is given by the following Hamiltonian,

$$
\mathcal{H}_{\mathcal{L}}=-J_{z} \sum_{\mu} \sigma_{\mu, L}^{z} \sigma_{\mu, R}^{z}
$$



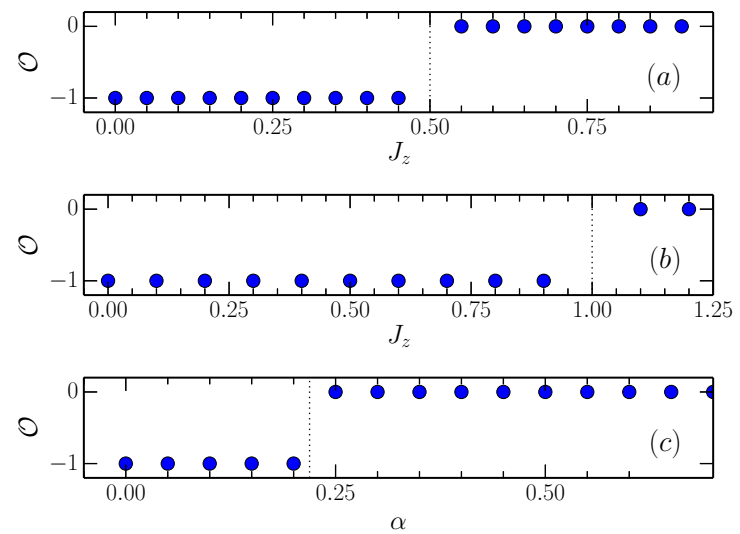

FIG. 9. (color online) Phase factor order parameter for ferromagnetic Kitaev ladder in the presence of (a) RI, (b) LI and (c) RLI interactions.

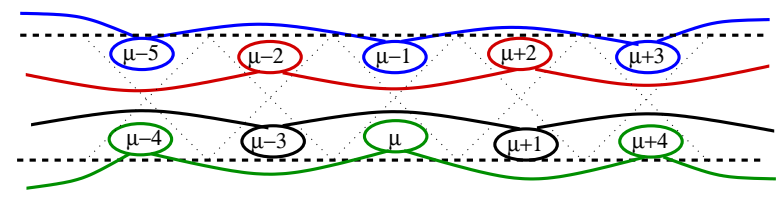

FIG. 10. (color online) Schematic representation of the interactions in the effective Hamiltonian of Kitaev ladder in the presence of LegIsing interactions. The model is equivalent to four decoupled TFI chains.

We explain the effect of $\sigma_{\mu, R}^{z} \sigma_{\mu, L}^{z}$ on quasi-spins. The quasispin associated by $\mu$ is not changed by this Ising term as it flips two spins, which leaves the product of spins on the triangle (quasi-spin) unchanged. However, the quasi-spins labeled by $\mu-3$ and $\mu+1$ are being flipped (see Fig. 2), which initiates the following correspondence in terms of quasi-spin operators,

$$
\sigma_{\mu, R}^{z} \sigma_{\mu, L}^{z} \longrightarrow \tau_{\mu-3}^{x} \tau_{\mu+1}^{x}
$$

In accordance with Eq 17 the Ising interactions along the legs are responsible for the interactions between the quasi-spins labeled $\bmod (4, n)$, independently, where $n=0,1,2,3$. Therefore, the effective Hamiltonian is described by four decoupled TFI chains, namely

$$
\mathcal{H}_{\text {eff }}^{\mathcal{K} \mathcal{L}}=-J_{z} \sum_{\mu}^{\prime \prime} \tau_{\mu}^{x} \tau_{\mu+4}^{x}-J_{v} \sum_{\mu}^{\prime \prime} \tau_{\mu}^{z},
$$

where $\sum_{\mu}^{\prime \prime}$ indicates four decoupled chains as shown in Fig 10. The effective model shows a quantum phase transition at $J_{z}=J_{v}$.

The quantum phase transition at $J_{z}=1$ (for $J_{v}=1$ ) is justified by von Neumann entropy versus $J_{z}$ plotted in Fig. 11 .

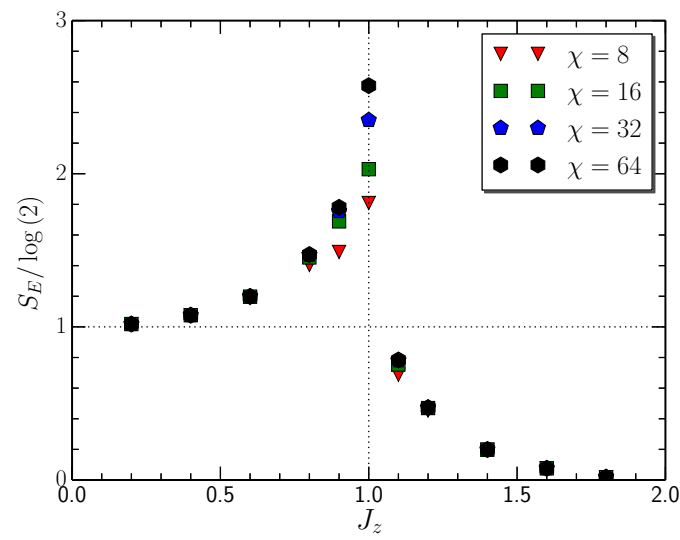

FIG. 11. (color online) von Neumann entropy versus $J_{z}$ for the Kitaev ladder with leg-Ising interactions. The entropy diverges exactly at $J_{z}=1$ confirmed by the effective theory $\left(\mathcal{H}_{\text {eff }}^{\mathcal{K} \mathcal{L}}\right)$.

For the small Ising coupling $\left(J_{z} \rightarrow 0\right) S_{E}$ is equal to $\ln (2)$ confirming the SPT phase of the pure Kitaev ladder. The entropy rises up and becomes divergent at $J_{z}=1$, which is the signature of quantum phase transition. Increasing the value of $J_{z}>1$ leads to a ferromagnetic phase for the original spins ordered in z-direction and mediated by the Ising interactions along the legs of ladder. The factorized ferromagnetic state gives a zero value for $S_{E}$ as is shown in Fig. 11 for the strong Ising coupling $\left(J_{z} \rightarrow \infty\right)$.

Another indication of quantum phase transition is found in the structure of entanglement spectrum and specially the degeneracy of levels. The degeneracy of the entanglement spectrum is even for $J_{z}<1$ while it becomes odd for $J_{z}>1$ (not shown here). The type of spectrum is similar to Fig. (4 except the change of degeneracy, which occurs at $J_{z}=1$. The even degeneracy for $J_{z}<1$ is a signature of the Kitaev SPT phase, which is verified by $\mathcal{O}=-1$ in Fig. 9 (b). The phase factor order parameter $(\mathcal{O})$ jumps to zero for $J_{z}>1$.

Our numerical results show that the quantum critical point between Kitaev SPT and ferromagnetic leg-Ising phase is described by the central charge $c=2.01 \pm 0.03$ as shown in Fig. 8-(b). This is in agreement with the effective theory obtained in Eq18, which shows four decoupled TFI chains that give $c=4 \times 0.5=2$.

The phase diagram of the Kitaev ladder with antiferromagnetic leg-Ising interaction is the mirror image of the ferromagentic phase diagram with respect to $J_{z}=0$. In fact, the full Hamiltonian is invariant under the transformation $J_{z} \rightarrow-J_{z}$ and a $\pi$ rotation around $\mathrm{x}$-axis on the even (or odd) spins on the legs of ladder.

\section{Rhombic-Leg-Ising interactions}

We consider the Ising interactions along the legs and the rhombic plaquettes that leads to more interesting phase dia- 


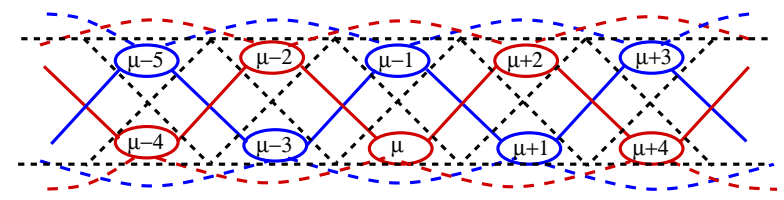

FIG. 12. (color online) The effective interactions between the quasispins for the Kitaev ladder with both rhombic and leg Ising terms. The rhombic terms create two decoupled TFI chains, namely odd and even ones, where the leg terms add the next-nearest neighbor interactions on each chain, separately. Solid-red (blue) lines represent the nearest neighbor interactions, while dashed-red (blue) line show the next-nearest neighbor ones, on even (odd) quasi-spins.

gram, where the full Hamiltonian is given by

$$
\mathcal{H}_{\mathcal{K} \mathcal{R} \mathcal{L}}=(1-|\alpha|) \mathcal{H}_{\mathcal{K}}+\alpha\left(\mathcal{H}_{\mathcal{R}}+\mathcal{H}_{\mathcal{L}}\right), \quad|\alpha| \leq 1
$$

Here, we introduce $\alpha$ to sweep between the extreme limits of Kitaev interaction for $\alpha=0$ and Ising limit for $|\alpha|=1 . \alpha>$ 0 corresponds to the ferromagnetic Ising interactions, while $\alpha<0$ represents the anti-ferromagnetic ones. The ground state phase diagram can be understood in terms of competition between the nearest and next-nearest neighbors interactions, which comes out of the effective theory. The antiferromagnetic Ising interactions have specific features, where frustration hinders simultaneous minimization of energy according to a classical antiferromagnetic state. The effective theory is simply obtained by incorporating the representation of rhombic-Ising and leg-Ising interactions in the quasi-spin representations. The Ising terms on rhombus lead to Ising interaction between even (odd) quasi-spins, while the leg terms establish interactions between quasi-spins of $\mu$ and $\mu+4$. Hence, the even and odd chain of quasi-spins remain decoupled bearing the next-nearest neighbor interactions, which are the effect of leg-Ising interactions. This can be seen in Fig. 12, where solid-red (blue) lines show NN and dashed-red (blue) lines represent NNN interactions for the even (odd) decoupled effective chains. It should be mentioned that the strength of NNN coupling is half of the NN one. The effective Hamiltonian for the Kitaev ladder in the presence of rhombic-leg-Ising interactions is given by

$$
\begin{aligned}
\mathcal{H}_{\text {eff }}^{\mathcal{K R} \mathcal{L}}= & -\alpha J_{z} \sum_{\mu}^{\prime}\left(2 \tau_{\mu}^{x} \tau_{\mu+2}^{x}+\tau_{\mu}^{x} \tau_{\mu+4}^{x}\right) \\
& -(1-|\alpha|) J_{v} \sum_{\mu}^{\prime} \tau_{\mu}^{z}, \quad \mu=\text { odd or even }
\end{aligned}
$$

The presence of NNN interactions in the antiferromagnetic regime lead to the interesting and exotic features in the model. Thus, we discuss the ferromagnetic and antiferromagnetic cases in the following two subsections, separately.

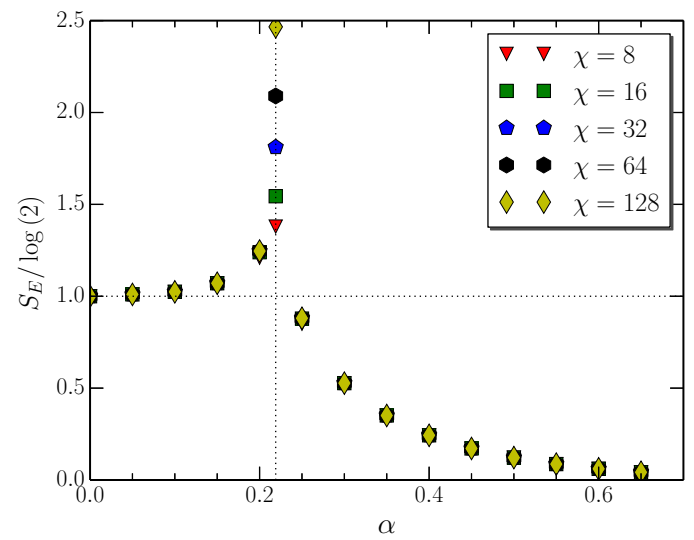

FIG. 13. (color online) von Neumann entropy (scaled by $\ln (2)$ ) versus $\alpha$ for the Kitaev ladder in the presence of ferromagnetic rhombicleg Ising interactions.

\section{Ferromagnetic RL-Ising}

Contrary to the RI and LI cases, the effective theory for the Kitaev ladder in the presence of both leg and rhombic Ising terms does not have an exact solution due to the NNN coupling in the TFI effective chain. Our numerical simulation of entropy, $S_{E}$ versus $\alpha$ is plotted in Fig. 13] which shows divergent behavior at the critical point $\alpha_{c}^{\mathcal{K R \mathcal { L }}}=0.219 \pm 0.001$. This is equivalent to a phase transition at $\left(J_{z} / J_{v}\right)=0.280 \pm$ 0.001 with a rescaling of the Kitaev and Ising couplings in Eq. 19. Here, the presence of Ising interactions on all bonds (legs and rhombuses) sustain the ferromagnetic order to overcome the Kitaev SPT phase within smaller $J_{z}$ coupling than the RI and LI cases. The model represents the Kitaev SPT

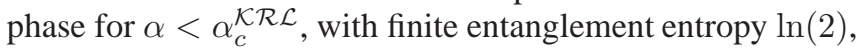
double degeneracy in the lowest entanglement spectrum, no (local) magnetic order, and phase factor order parameter $\mathcal{O}=$ -1 in Fig. 9.(c). A second order phase transition drives the

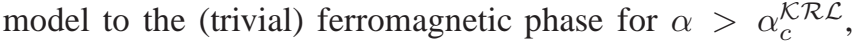
which is presented by a factorized state of up (or down) spins in z-direction. The quantum critical point is described by the central charge $c=2 \times 0.5=1$ given by two decoupled NNN TFI chain and justified by numerical simulation in Fig. 8. (c), which renders $c=1.09 \pm 0.02$.

\section{Antiferromagnetic RL-Ising}

The antiferromagnetic Ising interactions on both legs and rhombus bonds create the basic building block of frustrated magnetic systems, i.e., triangles with antiferromagnetic bonds (see Fig. 2). The antiferromagnetic Hamiltonian is defined by Eq. 19 with $-1 \leq \alpha \leq 0$. Although the ground state of the model is two-fold degenerate at the Kitaev limit $(\alpha=0)$ it has exponentially degenerate ground state configurations at 

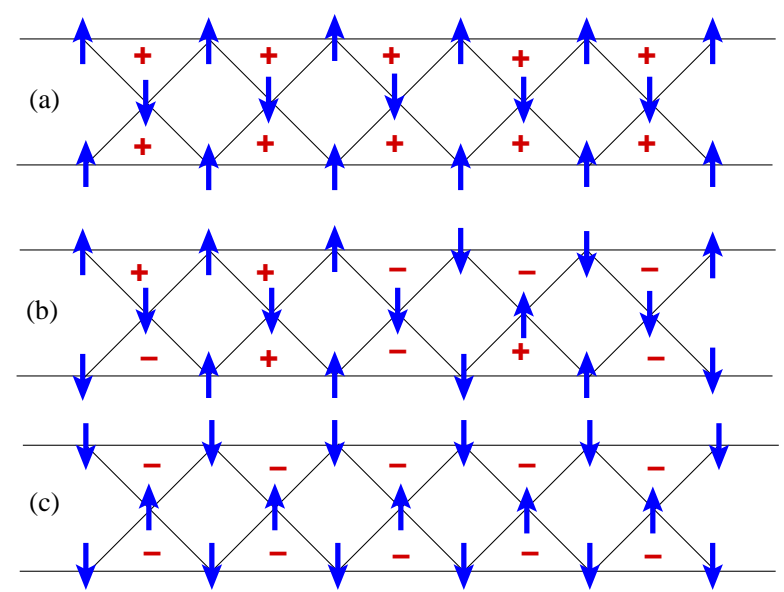

FIG. 14. (color online) Some of ground-state configurations at the antiferromagnetic Ising limit $\left(J_{v}=0\right)$ of the two-legs ladder. The $\uparrow$ and $\downarrow$ represent $\sigma^{z}$ spin orientation. The \pm shows the sum of $\sigma^{z}$ spins in each triangle. All configurations are classified as 2-up-1-down or 2-down-1-up for each triangle.

the AF Ising limit $(\alpha=-1)$. To get more insight on the model at the AF Ising limit, we associate a magnetization $\left(m_{\mu}^{z}\right)$ to each triangle, which is simply the total magnetization in $\mathrm{z}$-direction of a single triangle. For the ground state, the antiferromagnetic nature of interactions enforce the spins on each triangle to be oriented as either 2-up-1-down or 2down-1-up (see Fig. 14, which yields $m_{\mu}^{z}= \pm 1$. Therefore, the ground-state degeneracy at the AF Ising limit is $2^{2 N}$, where $2 N$ is the number of triangles in the ladder (assuming periodic boundary condition along legs). The spins that sit on the legs of ladder are not constraint to a boundary condition perpendicular to the legs of ladder, which leads to an intensive degenerate configurations with total magnetization $M^{z} \in\{2 N, 2 N-1, \ldots,-2 N+1,-2 N\}$,

$$
M^{z}=\sum_{\mu=1}^{2 N} m_{\mu}^{z} .
$$

Some configurations of the mentioned subspace are shown in Fig. 14, where the \pm in each triangle represents $m_{\mu}^{z}$. A state with $M^{z}=2 N$ is shown in Fig. 144-(a), where all triangles carry $m_{\mu}^{z}=+$, an intermediate state with $M^{z}=0$ is presented in Fig. 14.(b) and a state of all $m_{\mu}^{z}=-$ is given in Fig.14.(c). Accordingly, the model does not show a magnetic long-range order out of a symmetry breaking, which is called a classical spin-liquid.

We have plotted $S_{E}$ versus $\alpha$ in Fig. 15 for Kitaev ladder in the presence of AF RL-Ising interactions. Our data shows that $S_{E}$ reaches $\ln (2)$ for $\alpha \rightarrow 0$, which is the signature of Kitaev SPT phase. The entanglement entropy shows finite entanglement scaling for $-1<\alpha<-0.7$, which is represented by $\chi=8,16,32,64,128$. Although a bump is observed around $\alpha \simeq-0.8$ the whole set of data does not conclude to a single divergent peak, rather showing a broad area of finite entan- glement scaling. It suggests a broad critical area, which starts at $\alpha=-1$ (the classical spin-liquid) toward an intermediate region, $\alpha \simeq-0.75$, where the Kitaev SPT phase dominates. This is confirmed by the structure of entanglement spectrum versus $\alpha$ presented in Fig. 16 We have shown in Sec. [1 that the ground state of Kitaev ladder is an SPT phase, which leads to even degeneracy of ES. Accordingly, the even degeneracy of ES is the signature of Kitaev SPT phase for $\alpha_{c}<\alpha \leq 0$, where $\alpha_{c}=-0.75 \pm 0.05$. The computation for higher values of $\chi(>128)$ is a massive time consumption for our model, where the unit cell contains three spin-1/2. However, the results shown in Fig. 16 for $\chi=64$ (left), $\chi=128$ (right) and other $\chi=16,32$ (not shown here) convince us that the degeneracy of Kitaev SPT is persistent for $\alpha_{c}<\alpha \leq 0$. For $-1<\alpha \leq \alpha_{c}$, the model shows finite entanglement scaling (Fig. 15) and dispersed ES (Fig. 16), which resembles a critical area with algebraic decay of correlation functions. This is consistent with the conclusion that can be derived from the effective theory.

The effective Hamiltonian defined in Eq20 for Kitaev ladder in the presence of AF RL-Ising interactions renders the frustrated NNN TFI chain in which the NNN coupling is half of the NN one, being denoted by $\kappa=0.5$. The effective theory at $\alpha=-1$ falls exactly on the critical point $\kappa=0.5$ at zero transverse field, which separates the antiferromagnetic phase from the anti-phase of frustrated NNN TFF $36,39,40,42$. It states that the classical spin-liquid of the AF RL-Ising limit corresponds to the critical point of frustrated NNN TFI model at zero transverse field. The onset of Kitaev term $(\alpha \neq-1)$ adds quantum fluctuations to the model, which corresponds to the effect of transverse field on the frustrated NNN TFI critical point. A recent study on the frustrated NNN TFI chain ${ }^{42}$ confirms the existence of a tri-critical point at $\kappa=0.5$, where a Kosterlitz-Thouless transition line and two second order transition line merge at $\kappa=0.5$ and zero field. Thus, the effect of Kitaev term on the classical spin-liquid is similar to the effect of transverse field on frustrated NNN TFI at $\kappa=0.5$ toward passing through the floating phase before reaching a paramagnetic phase. The floating phase has algebraic decaying correlation functions, which could lead to finite entanglement scaling and broad dispersion of ES. Therefore, our results in Figs. 15, 16 are in agreement with the phase diagram proposed in Refs. 40 and 41 for NNN TFI (that is usually denoted by ANNNI model in the literature).

\section{TWO DIMENSIONAL KITAEV-ISING MODEL}

The extension of our model to two dimension would lead to the Kitaev toric code Hamiltonian ${ }^{\frac{5}{2}}$ in the presence of twobody Ising interactions. The toric code is defined on the twodimensional square lattice, where the spins sit on the bonds of lattice (filled-black circles in Fig. 17.(a)). The toric code Hamiltonian $\left(\mathcal{H}_{T}\right)$ is composed of two terms, vertex and pla- 


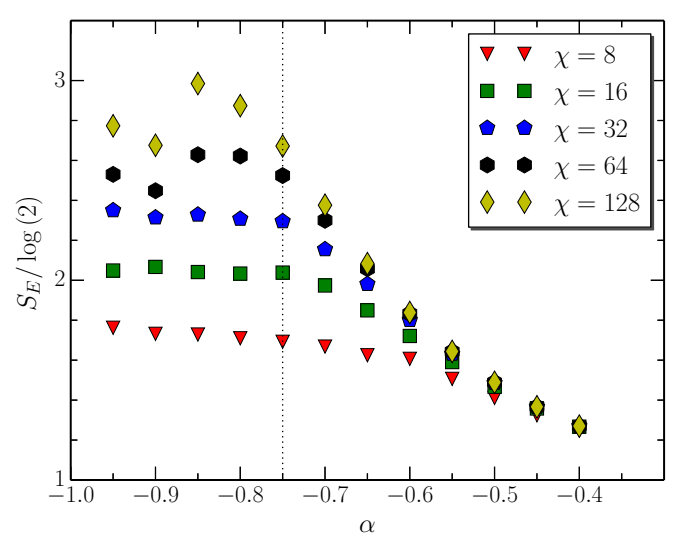

FIG. 15. (color online) von Neumann entropy versus $\alpha$ for the Kitaev ladder in the presence of AF RL Ising interactions.
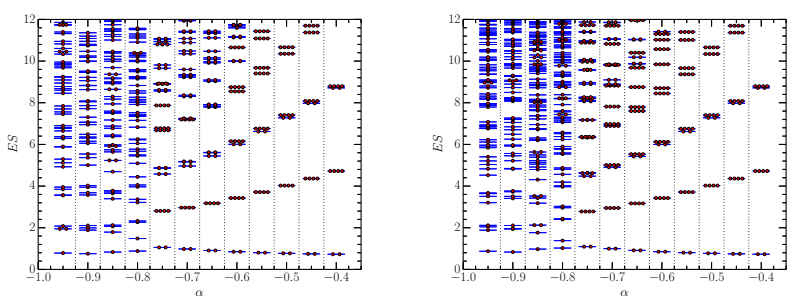

FIG. 16. (color online) Entanglement spectrum versus $\alpha$ for the Kitaev ladder in the presence of AF RL Ising interactions. (Left): $\chi=64$, (right): $\chi=128$.

quette ones, similar to Eq1,

$$
\mathcal{H}_{T}=-J_{v} \sum_{+} A_{v}-J_{p} \sum_{\square} B_{p}
$$

while both $A_{v}$ and $B_{p}$ are four-body interactions of $\sigma^{x}$ and $\sigma^{z}$ operators around each vertex and plaquette, respectively. The Ising interaction is composed of two-body interactions $-J_{v} \sigma_{i}^{z} \sigma_{j}^{z}$, where $(i, j)$ represent the nearest neighbor spins (filled-black circles in Fig.17-(a)). Similar to the ladder cases, we consider three types of Ising interactions, A: diagonal (D), where $(i, j)$ show the bonds along diagonal directions (on the rhombic shapes of Fig. 17.(a)), B: horizontal-vertical (HV) that defines the Ising bonds only on the horizontal and vertical links, C: full-Ising (FI), in which the Ising interactions exist on all diagonal, horizontal and vertical bonds of the model. The toric code in the presence of diagonal-Ising terms has been studied by Karimipour, et.al $\stackrel{21}{ }$, where a non-local transformation of bases map the model into a TFI model on a square lattice for two decoupled sublattices. Moreover, a mean-field approximation predict a quantum phase transition at $J_{v}=8 J_{z}$. Here, we implement the quasi-spin transformation, which creates the opportunity to study not only the diagonal-Ising case, but also the other two cases, namely: HV and full-Ising mod- els. It is very important to mention that the full-Ising interaction introduces a frustrated magnetic system, which would give rise to exotic phases.

The quasi-spin representation for the two-dimensional model is defined on each vertex, where a vertex operator $A_{v}=\sigma_{1}^{x} \sigma_{2}^{x} \sigma_{3}^{x} \sigma_{4}^{x}$ results in either +1 or -1 in the $\sigma^{x}$ representation. (The indices $1,2,3,4$ represent the four spins sharing a vertex). Thus, a quasi-spin is associated to this twovalued operator, which is called $T^{z}$,

$$
A_{v} \longrightarrow T^{z} .
$$

As before, the plaquette term $\left(B_{p}\right)$ commutes with both vertex and any types of Ising interaction. Therefore, $B_{p}$ does not participate in the competition of a quantum phase transition and is being kept to its minimum value to insure being in the low-energy sector. Hence, the toric code Hamiltonian is represented by a magnetic field within the quasi-spin representation,

$$
\mathcal{H}_{T}=-J_{v} \sum_{+} A_{v} \longrightarrow-J_{v} \sum_{\nu} T_{\nu}^{z},
$$

where $\nu$ runs over all vertices of the two-dimensional lattice. An Ising interaction flips the value of a quasi-spin if it shares one spin with the vertex and do nothing if it shares two spins with the vertex. Based on the type of Ising interaction we find different effective Hamiltonians for $\mathcal{H}_{2 D}=\mathcal{H}_{T}+\mathcal{H}_{\text {Ising }}$, which are given in the following subsections.

\section{A. Diagonal Ising interactions}

The diagonal-Ising interaction does not change the state of a vertex that shares two spins while it flips the state of two vertices (along diagonal direction) that share only one spin. The right upper part of Fig. 17. (a) shows two quasi-spins denoted by blue circles, which are being flipped according to the diagonal-Ising terms (shown by diagonal-solid black lines). The effective interaction is represented by the solid-blue line in the diagonal direction. Moreover, the four vertices around a rhombus are decomposed to two decoupled sublattices shown by blue and red circles. Hence, the effective Hamiltonian for the toric code in the presence of diagonal-Ising interactions is given by

$$
\mathcal{H}_{e f f}^{T D}=-2 J_{z} \sum_{<\nu, \nu^{\prime}>} T_{\nu}^{x} T_{\nu^{\prime}}^{x}-J_{v} \sum_{\nu} T_{\nu}^{z},
$$

where $\left\langle\nu, \nu^{\prime}\right\rangle$ runs on the nearest neighbor quasi-spins of either blue or red sublattices. The lattice of quasi-spins (either blue or red) is a two-dimensional square lattice rotated $\pi / 4$ with respect to the original lattice, namely shown by the $x-y$ unit vectors in Fig. 177-(a). This is in complete agreement with the effective model presented in Ref 21 , which shows a quantum phase transition from the $\mathbb{Z}_{2}$ spin-liquid ground state of topological toric code $\left(J_{z}=0\right)$ to a ferromagnetic product state of Ising limit $\left(J_{v}=0\right)$. According to the Monte-Carlo ${ }^{54}$ and DMRG 5 simulations on 2D TFI model, the quantum critical point is at $J_{v}=6.09 J_{z}$. Similar behavior is expected for 


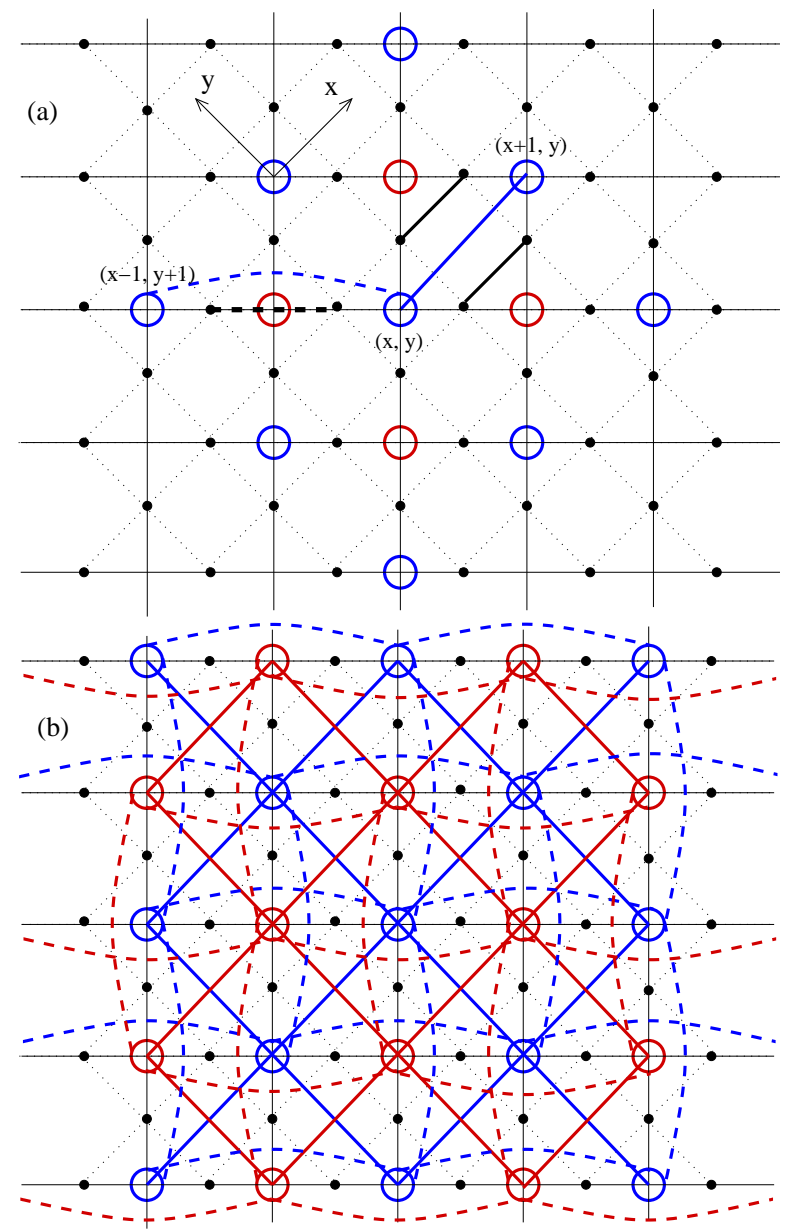

FIG. 17. (color online) (a) Diagonal-Ising interactions (solid-black lines in the upper right part) and its corresponding effective interaction on quasi-spins (solid-blue line). Horizontal-Ising interaction (dashed-black line in the left part) and its corresponding effective interaction on quasi-spins (dashed-blue line). Blue and red circles represent the quasi-spins. (b) Two sublattices (blue and red), which interact like TFI model with next-nearest neighbor interactions as an effective theory to describe the toric code Hamiltonian in the presence of full-Ising interactions.

the antiferromagnetic diagonal-Ising interactions, which can be obtained by the transformation $J_{z} \rightarrow-J_{z}$ and $\sigma_{i}^{z} \rightarrow-\sigma_{i}^{z}$ for $i \in \Sigma_{A}$, where $\Sigma_{A}$ is one of the sublattices of the original bipartite two-dimensional lattice. Needless to mention that the AF Ising limit presents a Néel ordered state.

\section{B. Horizontal-Vertical Ising interactions}

A bond of horizontal (or vertical) Ising interaction does not change the state of a quasi-spin, which shares two spin at the corresponding vertex. This is shown by the tick-dashed-black line in the left part of Fig. 17.(a), which crosses a red circle. The quasi-spin denoted by the red circle is not changed, while the quasi-spins of the horizontal neighboring blue circles are being flipped. Hence, a horizontal (vertical) Ising interaction flips the nearest neighbor quasi-spin of a sublattice oriented horizontally (vertically). The lattice of quasi-spins denoted by blue and red circles in Fig. 17. (a) is bipartite, where the blue and red circles represent the two sublattices. Thus, the effective model of toric code with HV-Ising interactions is given by the following Hamiltonian

$$
\mathcal{H}_{\text {eff }}^{T H V}=-J_{z} \sum_{<\nu, \nu^{\prime}>} T_{\nu}^{x} T_{\nu^{\prime}}^{x}-J_{v} \sum_{\nu} T_{\nu}^{z},
$$

in which $\left\langle\nu, \nu^{\prime}\right\rangle$ defines nearest neighbor quasi-spins of either blue or red sublattices. Each sublattice forms a twodimensional square lattice where the lattice constant is twice as the original one. According to Ref 54, the QPT takes place at $J_{v}=3.044 J_{z}$ from the $\mathbb{Z}_{2}$ spin-liquid state $\left(J_{z}=0\right)$ to the ferromagnetic product state. Similar to the previous case, the phase diagram is symmetric with respect to $J_{z} \rightarrow-J_{z}$, which covers the antiferromagnetic regime. A remark is in order here, the original (and also the effective lattice) is bipartite for both D-Ising and HV-Ising interactions at the extreme limit of Ising interactions $\left(J_{v}=0\right)$. This avoids frustration for the antiferromagnetic Ising couplings, which is not preserved for the full-Ising interactions as will be discussed next.

\section{Full-Ising interactions}

The effective theory of toric code in addition to full-Ising interactions can be obtained by considering the representations of both diagonal and HV-Ising interactions in the quasispin subspace. The following facts help to realize easily the structure of the effective theory. (i) The quasi-spin lattice (of blue and red circles) is bipartite such that neither a diagonal nor an HV-Ising interaction make an interaction between the blue and red sublattices. (ii) The quasi-spin representation forms a square lattice whose lattice spacing is $\sqrt{2}$ times larger than the original one and is rotated $\pi / 4$ with respect to the original lattice. The unit-vectors of the quasi-spin lattice are labeled by $x-y$ in Fig. 17-(a). (iii) The diagonal-Ising interactions establish the nearest neighbor interactions of strength $2 J_{z}$ on each sublattice. (iv) The HV-Ising interactions build up the next-nearest neighbor (diagonal) interactions of strength $J_{z}$ on each sublattice. Accordingly, the effective model of the toric code in the presence of full-Ising interactions is

$$
\begin{gathered}
\mathcal{H}_{e f f}^{T F I}=-2 J_{z} \sum_{<\nu, \nu^{\prime}>} T_{\nu}^{x} T_{\nu^{\prime}}^{x}-J_{v} \sum_{\nu} T_{\nu}^{z} \\
-J_{z} \sum_{<<\nu, \nu^{\prime}>>} T_{\nu}^{x} T_{\nu^{\prime}}^{x},
\end{gathered}
$$

where $\left\langle\nu, \nu^{\prime}>\right.$ stands for $\mathrm{NN}$ and $\left\langle<\nu, \nu^{\prime}>>\right.$ for NNN quasi-spins. The effective Hamiltonian is a two-dimensional NNN TFI model on a square lattice that is shown in Fig. 17 . (b), where the blue and red colors show two decoupled sublattices. The solid lines show NN interactions while the dashed one show NNN ones. 
In the ferromagnetic regime $\left(J_{z}>0\right)$ a quantum phase transition occurs from the topological toric code ground state $\left(J_{z}=0\right)$ to the symmetry broken ferromagnetic state. The corresponding QCP is at $J_{v}=12 J_{z}$ within the mean-field approximation.

The antiferromagnetic regime, $J_{z}<0$, would be essentially different from the ferromagnetic one as a result of frustration in the $\mathrm{AF}$ Ising limit $\left(J_{v}=0\right)$. The frustration arises from the antiferromagnetic bonds, which form triangles in the original lattice. The ground-state manifold is highly degenerate, which is composed of triangles having 2-up-1-down or 1-up-2-down spins (in z-direction). This is a manifestation of classical spin-liquid phase on a two-dimensional lattice. It suggest a quantum phase transition from the classical spinliquid phase $\left(J_{v}=0\right)$ to the $\mathbb{Z}_{2}$ topological spin-liquid phase $\left(J_{z}=0\right)$ at a finite ratio of $J_{z} / J_{v}$. However, according to the effective theory presented in Eq27, we expect an orderby-disorder phase transition before the transition to the topological spin-liquid phase. At the AF Ising limit $\left(J_{v}=0\right)$ the effective theory is known as $J_{1}-J_{2}$ Ising model on square lattice with $J_{2}=0.5 J_{1}$, where $J_{1}$ is NN and $J_{2}$ is NNN antiferromagnetic interactions. It is known that $J_{2}=0.5 J_{1}$ is the critical point of $J_{1}-J_{2}$ Ising model, which separates the Néel ordered phase $\left(J_{2}<0.5 J_{1}\right)$ from the collinear ordered one $\left(J_{2}>0.5 J_{1}\right)$. The critical nature of $J_{2}=0.5 J_{1}$ of our effective theory manifests the frustration induced by triangles of the original model, which leads to an extensive degeneracy in both the effective ground state and the original one. The onset of transverse magnetic field $(\Gamma)$ at the critical point $J_{2}=0.5 J_{1}$ of the effective theory leads to an order-by-disorder ${ }^{56}$, which looks like a ferromagnetic order for small fields, $\Gamma \lesssim 0.2 J_{1}$, before a transition to the fully polarized state. This is in agreement with the order-by-disorder transition proposed for the fully-frustrated Ising model on the square lattice 48 A ferromagnetic state of the effective theory, $\left\langle T_{\nu}^{x}\right\rangle=1$, corresponds to $A_{v}=1$, in which only those spin configurations of two-up-two-down on a vertex contribute to the ground state, which can be called a resonating ice-state resembling the ice-rule configuration of spin-ice. The spinice configurations do not include the all-up or all-down states of $A_{v}=1$ subspace. Hence, the classical spin-liquid state is unstable against quantum fluctuations at $J_{v}=0$ toward a resonating ice-state for $0<J_{v} \lesssim 0.2 J_{z}$. Increasing the vertex coupling makes a transition to the topological $\mathbb{Z}_{2}$ spin-liquid state for $J_{v} \gtrsim 0.2 J_{z}$. More investigations is required, which needs demanding resources for numerical computations that is beyond the scope of present manuscript.

\section{SUMMARY AND DISCUSSION}

We have studied the Kitaev Hamiltonian $\left(\mathcal{H}_{\mathcal{K}}\right)$ on a ladder geometry. We find that the ground state of Kitaev ladder is an SPT phase protected by $\mathbb{Z}_{2} \times \mathbb{Z}_{2}$ symmetries namely $\mathcal{X}=\prod_{i} \sigma_{i}^{x}$ which runs over all ladder bonds and $\mathcal{Z}=$ $\prod_{\ell \notin \text { rungs }} \sigma_{\ell}^{z}$ that excludes the rung bonds. We have justified our argument by employing iDMRG method within an iMPS representation, which leads to inequivalent projective representation of $\mathbb{Z}_{2} \times \mathbb{Z}_{2}$ symmetries providing the phase factor order parameter $\mathcal{O}=-1$, for the Kitaev phase.

We have also investigated the competition between the Kitaev and Ising terms on the ladder, which is given by deriving the corresponding effective theory in addition to the direct iDMRG computations. For the Ising interactions being solely on the edges of rhombus or on the legs, the effective Hamiltonian is given by decoupled one-dimensional TFI models, which explains the quantum phase transition from the Kitaev SPT phase to the antiferro/ferro-magnetic phase at the exact finite value $J_{z} / J_{v}=0.5,1.0$, respectively. The quantum phase transition is justified by numerical divergence of entanglement entropy $\left(S_{E}\right)$ at the critical point, the change in the degeneracy of the entanglement spectrum, ground-state fidelity, and magnetic order parameters. The quantum critical points and their corresponding central charges of the effective theory and iDMRG results agree with each other, exactly. The critical behavior of Kitaev-rhombic-Ising interactions is given by central charge $c=1$, while the Kitaev-leg-Ising ladder is represented by $c=2$. If the Ising interactions reside on both rhombus and legs of ladder, the effective theory would be NNN TFI chain with the critical properties given by $c=1$. For the ferromagnetic Ising interactions it leads to a quantum phase transition at finite ratio $J_{z} / J_{v}=0.28$, while for the antiferromagnetic Ising interactions our data shows a broad range of finite entanglement scaling. It is the interplay between the classical spinliquid at AF Ising limit and the Kitaev SPT phases. According to the effective theory, the onset of Kitaev term induces quantum fluctuations in the classical spin-liquid subspace, which would finally lead to the Kitaev SPT phase passing through an intermediate floating phase. The Kitaev SPT phase is persistent for $\left|J_{z}\right| / J_{v} \lesssim 3$ witnessed by the even degeneracy of the entanglement spectrum.

A remark is in order concerning the effective theory introduced in this paper. For simplicity, we consider the ladder geometry with periodic boundary condition along the legs, where $N$ is the number of spins sitting on each leg or rung of ladder, which sums up to $3 N$ spins. The dimension of Hilbert space of the original ladder is $2^{3 N}$. The number of triangles (quasi-spins) is $2 N$ and the number of rhombuses (plaquettes) is $N$. Accordingly, the dimension of the Hilbert space of the effective theory (in terms of quasi-spins) is $2^{2 N}$ which is smaller than the original Hilbert space by a factor of $2^{N}$. In principle, this is always the case for an effective theory, which is responsible for the low-energy behavior of the original model and is confirmed by numerical iDMRG results. However, taking into account the plaquette degrees of freedom $\left(B_{p}= \pm 1\right)$ we find the lost $2^{N}$ degrees of freedom. For the lowest energy spectrum we consider all $N$-plaquettes to be at $B_{p}=+1$, which adds a constant term $-J_{p} N$ to the effective theory. As far as all configurations of the original spin model have been kept in constructing the effective theory we expect that the whole spectrum of the original model is represented by a tower of TFI models in addition to their corresponding constant values, i.e., $\left(-J_{p}\right) \sum_{p} B_{p}$.

Having in mind that the effective theory considers the whole degrees of freedom of the original model, we find the 
exact critical exponents of the mentioned QCPs. For instance, we calculate in detail the magnetization exponent $(\beta)$ close to transition of the ferromagnetic phase of Kitaev RI-ladder (Sec. III A). The ferromagnetic order parameter $\left\langle\sigma_{z}\right\rangle$ is

$$
\left\langle\sigma_{z}\right\rangle=\frac{1}{3 N} \sum_{i=1}^{N}\left\langle\psi_{0}\left|\sigma_{1}^{z}(i)+\sigma_{3}^{z}(i)+\sigma_{3}^{z}(i)\right| \psi_{0}\right\rangle .
$$

The effect of $\sigma_{1}^{z}(i)$ on the ground state of ladder $\left(\left|\psi_{0}\right\rangle\right)$ is equivalent to flip the state of quasi-spins denoted by the two triangles, which share $\sigma_{1}^{z}(i)$ at their common corner. It is shown by

$$
\sigma_{1}^{z}(i)\left|\psi_{0}\right\rangle=\tau_{\mu}^{x}\left|\varphi_{0}^{(e v e n)}\right\rangle \otimes \tau_{\mu-1}^{x}\left|\varphi_{0}^{(o d d)}\right\rangle,
$$

where $\left|\varphi_{0}^{(\text {even })}\right\rangle\left(\left|\varphi_{0}^{(\text {odd })}\right\rangle\right)$ represents the ground state of TFI effective theory for even (odd) decoupled chain. Hence, we conclude that

$$
\left\langle\sigma_{z}\right\rangle=\left(\left\langle\tau^{x}\right\rangle_{T F I}\right)^{2}=\left|J_{z}-J_{z}^{c}\right|^{\frac{1}{4}},
$$

which leads to $\beta=1 / 4$ as confirmed numerically in the inset of Fig.7. A similar calculation gives the exponent of the algebraic decay of correlation functions at the QCP,

$$
\left.C(r)\right|_{J_{z}=J_{z}^{c}}=\left\langle\sigma_{1}^{z}(i) \sigma_{1}^{z}(i+r)\right\rangle \sim \frac{1}{r^{\eta}}, \quad \eta=\frac{1}{2} .
$$

This is in agreement with the numerical computation of correlation function of the Kitaev RI-ladder performed at $J_{z}=$ $J_{z}^{c}=0.5$ in Fig. 18, where the numerical exponent is $\eta=$ $0.50 \pm 0.01$. It should be noticed that both $\beta$ and $\eta$ exponents are twice as the corresponding one of the TFI chain. It means that the decoupled chains of the effective theory contribute to the quantum critical properties of the original ladder. In other words, although the effective TFI chains are decoupled they are not independent. Our calculations for all critical points of AF/F Kitaev RI, LI and F RLI cases give the same exponents, namely $\beta=1 / 4$ and $\eta=1 / 2$. The case of AF RLI needs more delicate considerations being close to a Kosterlitz-Thouless transition, which needs more extensive numerical computations out of the scope of this article.

The extension of our approach to the two dimensional case would lead to study the competition between the topological $\mathbb{Z}_{2}$ spin-liquid state of the toric code with the symmetry broken or classical spin-liquid state of Ising interactions. If the Ising interactions exist either on the diagonal or horizontal-vertical direction of the two-dimensional lattice, the QPT between topological ground state of toric code and the ferro/antiferro-magnetic phase is given by the TFI model on two-dimensional square lattice. However, in the case of both diagonal and horizontal-vertical Ising interactions the effective theory is NNN TFI on 2D square lattice. For the ferromagnetic Ising interactions, it leads to a quantum phase transition at finite ratio $J_{v} / J_{z}$, while for the antiferromagnetic case we expect an order-by-disorder transition at $J_{v}=0$ followed by a transition to the spin-liquid state at $J_{v} / J_{z} \simeq 0.2$. The frustration induced by the antiferromagnetic coupling hinders

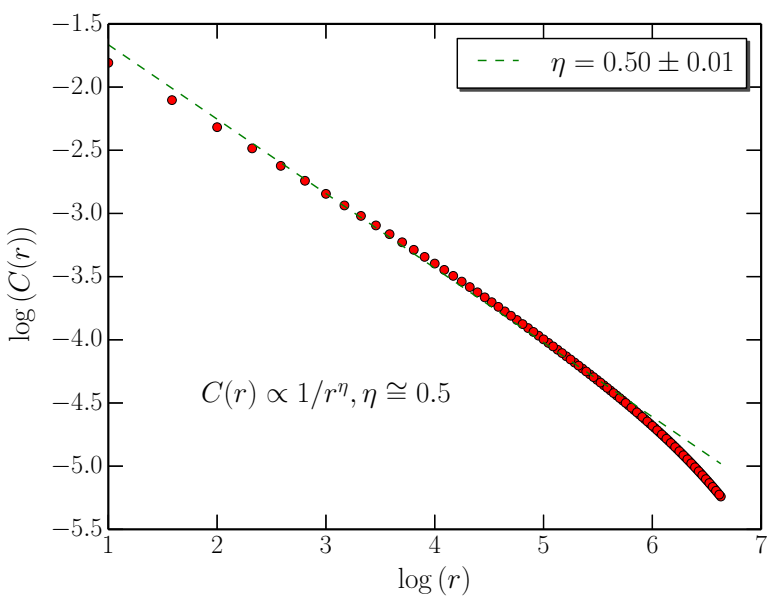

FIG. 18. (color online) Log-log plot of correlation function $C(r)$ versus $r$ for the Kitaev-rhombic Ising ladder at the QCP. The (green) dotted line shows the best fit of $r^{(-0.5)}$, which states $\eta=0.50 \pm 0.01$. The correlation length is $\xi \backsim 400$ for $\chi=150$, which determines the reliable behavior for $r<\xi$, i.e. $\log (r) \lesssim 5.99$.

the long-range order of the Ising limit toward an exponentially degenerate ground state configurations called classical spinliquid. According to the effective theory, the onset of Kitaev toric code term perturb the model toward an ordered phase for $J_{v} / J_{z} \lesssim 0.2$ and finally the $\mathbb{Z}_{2}$ spin-liquid state appears for $J_{v} / J_{z} \gtrsim 0.2$.

\section{ACKNOWLEDGMENTS}

The authors would like to thanks S. Bhattacharjee, J. Kjäll, S. Moghimi-Araghi, O. Petrova, F. Pollmann and A. T. Rezakhani for fruitful discussions and comments. This work was supported in part by the Office of Vice-President for Research of Sharif University of Technology. A. L. gratefully acknowledges the Alexander von Humboldt Foundation for financial support.

\section{Appendix A: Numerical approach: iDMRG}

To examine the properties of the model, we have made use of the standard iDRMG technique that is based on an infinite matrix product state (iMPS) representation for the ground state ${ }^{43,45}$. It is a well known fact that iMPS is an efficient method to describe translationally invariant many body states with an accuracy depending on the dimension of implemented matrices 57,58 .

The translationally invariant ground state is characterized by canonical ${ }^{\underline{59}} \Gamma$ and $\Lambda$ matrices,

$$
|\Psi\rangle=\sum_{s_{1}, \ldots, s_{N}} \operatorname{Tr}\left[\Gamma^{s_{1}} \Lambda \ldots \Gamma^{s_{N}} \Lambda\right]\left|s_{1} \ldots s_{N}\right\rangle,
$$


which satisfy the following (fixed point) relation,

$$
\sum_{s} \Gamma^{s} \Lambda^{s} \Lambda^{s \dagger} \Gamma^{s \dagger}=\mathbb{1}
$$

where the sum is over different spin configurations and $\Gamma \mathrm{s}$ serve as the matrix coefficients for these configurations.

The spectrum (i.e. singular values in the Schmidt decomposition of the left and right bipartition of the Hilbert space) is simply the square root of $\Lambda$ and the entanglement entropy is defined as,

$$
S_{\mathrm{E}}=-\sum_{i} \Lambda_{i i}^{2} \log \left(\Lambda_{i i}^{2}\right)
$$

In the general case, to give an exact representation of a state, iDMRG needs infinitely large matrices. Hopefully, this is not necessary, specially in the case of gapped systems, by putting an upper bound on the cardinality of the matrices $\chi$, and truncating the spectrum, one can reach a good approximation, which has all the properties of the low energy state. This will give rise to the so-called, truncation error which can be controlled by the dimension of matrices and is the cause of the entropy scaling.

Since the ground state is known to be gapped for the extreme coupling limit of our model, it can be represented by a finite iMPS, where the truncation error for the couplings that are far from the critical point, is less than machine precision. However, close to the critical point, when the ground state entanglement spectrum should show a long tail, the truncation errors become considerable and they do not vanish even when we increase the size of matrices.

After reaching the canonical $\Lambda, \Gamma$ with the desired accuracy, several properties of the ground state can be evaluated using the iMPS representation. It includes any local observable like energy and $\sigma^{z}$, entanglement spectrum and the corresponding von-Neumann entropy, the application of symmetry operators, and the ground state fidelity ${ }^{60}$. In order to calculate the mentioned quantities the concept of transfer matrix should be introduced,

$$
T_{\alpha \alpha^{\prime}, \beta \beta^{\prime}}=\sum_{s}\left(\Gamma_{\alpha \beta}^{s} \Lambda_{\beta}\right)\left(\Gamma_{\alpha^{\prime} \beta^{\prime}}^{s} \Lambda_{\beta^{\prime}}\right)^{*}
$$

Expectation value of a local operator (defined on one specific site), such as $\hat{\mathcal{O}}$, is obtained by using the following transfer matrix,

$$
\widehat{T}_{\alpha \alpha^{\prime}, \beta \beta^{\prime}}=\sum_{s, s^{\prime}}\left(\Gamma_{\alpha \beta}^{s} \Lambda_{\beta}\right) \hat{\mathcal{O}}^{s, s^{\prime}}\left(\Gamma_{\alpha^{\prime} \beta^{\prime}}^{s^{\prime}} \Lambda_{\beta^{\prime}}\right)^{*}
$$

Expectation value of $\langle\Psi|\hat{\mathcal{O}}| \Psi\rangle$ simply reduces to $\operatorname{Tr}(\Lambda \otimes \Lambda \widehat{T})$. For the fidelity calculations, iDMRG should calculate the canonical iMPS for two very close couplings $\left(J_{z}^{-}, J_{z}^{+}\right)$,

$$
\begin{gathered}
J_{z}^{-}=J_{z}-\delta / 2, \\
J_{z}^{+}=J_{z}+\delta / 2
\end{gathered}
$$

For an iMPS state the fidelity is defined as the largest eigenvalue of the transfer matrix constructed as the product of two close couplings,

$$
\bar{T}_{\alpha \alpha^{\prime}, \beta \beta^{\prime}}^{\delta}=\sum_{s}\left(\Gamma_{\alpha \beta}^{s} \Lambda_{\beta}\right)_{J_{z}^{-}}\left(\Gamma_{\alpha^{\prime} \beta^{\prime}}^{s} \Lambda_{\beta^{\prime}}\right)_{J_{z}^{+}}^{*}
$$

The central charge is calculated according to the scaling relation between von-Neumann entropy and the correlation length. The correlation length is defined as the second largest eigenvalue $\left(e_{2}\right)$ of the transfer matrix $T^{52,53}$,

$$
S_{\mathrm{E}} \propto \frac{c}{6} \log (\xi), \quad \xi=-\frac{1}{\ln \left(e_{2}\right)} .
$$

The general scheme of the algorithm for our model is as follows. First, we formulate the model to get a 1D model with only NN interactions, we bundle every three particles on a triangle, as shown in the Fig.1 into one unit-cell with dimension 8. For instance, according to the definition in Eq 3 the Kitaev Hamiltonian is written in the following form

$\mathcal{H}_{\mathcal{K}}=-J_{v} \sum_{<i, j>} \Sigma(i)^{I x I} \Sigma(j)^{x x I}-J_{p} \sum_{<i, j>} \Sigma(i)^{z z z} \Sigma(j)^{z I I}$.

Similar expressions would be used for the Ising terms. The CPU time of iDMRG algorithm is proportional to the square of the spin dimensions $d^{2}$. It is obvious that the calculation time needed to accomplish a simple iDMRG on the mentioned lattice (with $d=8$ ) is much larger than iDMRG performed on a lattice with spin dimension 2. This is the main reason that we were unable to examine larger matrices for this model. For example, the necessary time for convergence was about couple of weeks for a single run of matrix size $\chi=128$, and close to critical region. The convergence criterion was a fixed point relation between the $\Lambda$ generated at the current step with the $\Lambda$ of the last step.

We have also examined the iDMRG for one-dimensional NNN TFI model and compared the results with the corresponding one of the original ladder. The entanglement spectrum and hence the entropy was the same within relative error of $10^{-5}$.

\section{Appendix B: Symmetry}

A symmetry is defined as an operation, which leaves the model Hamiltonian invariant. These symmetries can form either an ordinary group or a projective one. However, if the ground state of Hamiltonian does not respect the Hamiltonian symmetries, one concludes the phase is a symmetry broken one. At the same time, the remaining symmetry groups can protect a phase due to their inequivalent projective representations, also known as symmetry fractionalization. These two properties can be used to assign a unique label to every possible phase of a system and to detect possible phase transition within this classification 28 .

The ground state of the Kitaev ladder Hamiltonian $\left(\mathcal{H}_{\mathcal{K}}\right)$ is doubly degenerate and both ground states are invariant under 
the operations of $\mathcal{X}$ and $\mathcal{Z}$ (defined in Eq4). The mutual symmetry operation $\mathcal{X} \times \mathcal{Z}$ defines a $\mathbb{Z}_{2} \times \mathbb{Z}_{2}$ symmetry, which protects the Kitaev ladder ground states. In other words, providing the symmetry is preserved, the Kitaev SPT phase can not be adiabatically mapped to a fully product state.

To gain more insight on how the $\mathbb{Z}_{2} \times \mathbb{Z}_{2}$ symmetry group can serve to protect the degeneracy of the entanglement spectrum, and how we can express them numerically we need to explore the properties of the symmetry group in terms of iMPS representation. To preserve the $\mathbb{Z}_{2} \times \mathbb{Z}_{2}$ symmetry for an iMPS state, the following relation should be satisfied $\underline{61}$,

$$
\sum_{s^{\prime}} u_{s s^{\prime}}(g) \Gamma_{\alpha \alpha^{\prime}}^{s^{\prime}}=U^{\dagger}(g)_{\alpha \beta} \Gamma_{\beta \beta^{\prime}}^{s} U(g)_{\beta^{\prime} \alpha^{\prime}}
$$

where $u(g) \in G, G=\left\{\Sigma^{I z z}, \Sigma^{x x x},-\Sigma^{x y y}, \Sigma^{I I I}\right\}$ and $g$ represents the index of group elements. To obtain $U_{g}$ for all elements of the group $G$, we construct the following transfer matrix $\left(\widehat{T}^{g}\right)$ for each element of group $G$,

$$
\widehat{T}_{\alpha \alpha^{\prime}, \beta \beta^{\prime}}^{g}=\sum_{s, s^{\prime}}\left(\Gamma_{\alpha \beta}^{s} \Lambda_{\beta}\right) u(g)^{s, s^{\prime}}\left(\Gamma_{\alpha^{\prime} \beta^{\prime}}^{s^{\prime}} \Lambda_{\beta^{\prime}}\right)^{*}
$$

The symmetry represented by $u(g)$ on all sites is respected, if the largest eigenvalue of $\widehat{T}^{g}$ becomes equal to 1 . Using Eq. B1] one can show the corresponding eigenvector is simply $U_{g}^{\dagger}$.

Generally, $U_{g}, U_{g^{\prime}}$ may not always form a regular group but a projective one. To see this behavior we need to apply the symmetries in different order and make use of the facts that $u(g) u\left(g^{\prime}\right)=u\left(g^{\prime}\right) u(g)$ and $u^{2}(g)=\mathbb{1}$. Using $u(g) u\left(g^{\prime}\right)=$ $u\left(g^{\prime}\right) u(g)$, we conclude

$$
\begin{gathered}
u(g) u\left(g^{\prime}\right) \Gamma=U_{g} U_{g^{\prime}} \Gamma U_{g^{\prime}}^{\dagger} U_{g}^{\dagger}, \\
u\left(g^{\prime}\right) u(g) \Gamma=U_{g^{\prime}} U_{g} \Gamma U_{g}^{\dagger} U_{g^{\prime}}^{\dagger}, \\
\Rightarrow U_{g} U_{g^{\prime}}=e^{i \Omega_{g g^{\prime}} U_{g^{\prime}} U_{g},}
\end{gathered}
$$

where the phase $e^{i \Omega_{g g^{\prime}}}$ is called "phase factor" (for simplicity we drop indices corresponding to summations). The property of $u^{2}(g)=\mathbb{1}$ results in $U^{2}(g)=e^{i \theta_{g}} \mathbb{1}$. Using Eq. B3 and $U^{2}(g)=e^{i \theta_{g}} \mathbb{1}$, one can easily show that $e^{i \Omega_{g g^{\prime}}}$ can only be \pm 1 . The signs introduce two different kind of orders, i.e. SPT and trivial orders. Throughout the SPT (trivial) phase, $e^{i \Omega_{g g^{\prime}}}=-1(+1)$ and only upon quantum phase transition, the sign can change. The two signs also represent two inequivalent projective representations of $\mathbb{Z}_{2} \times \mathbb{Z}_{2}$ symmetry.

One can exploit this property and define an order parameter $\mathcal{O}$ (called phase factor order parameter), which can serve to detect, by measuring the sign, which projective representation holds for a possible phase,

$$
\mathcal{O}=\frac{1}{\chi} \operatorname{Tr}\left(U_{g} U_{g^{\prime}} U_{g}^{\dagger} U_{g^{\prime}}^{\dagger}\right)
$$

When the iMPS doesn't possess one of the symmetries in the group, the phase factor order parameter $\mathcal{O}$ is simply 0 , demonstrating symmetry broken phase.

The proposed $\mathbb{Z}_{2} \times \mathbb{Z}_{2}$ symmetry group is not just the symmetry for the Kitaev Hamiltonian, but it commutes with the Ising interactions as well. As a result the phase factor order parameter $\mathcal{O}$, can be a good quantity to observe the phase transition which kills the symmetry protected phase. When the system is close to the Kitaev phase the phase factor order parameter has a negative sign, which shows the system is in a symmetry protected state, but for the ferromagnetic phase, while $\Sigma^{I z z}$ is still respected, $\Sigma^{x x x}$ is no longer preserved and the phase operator order parameter suddenly drops to zero, as shown in Fig. 9
* langari@ sharif.edu, http://sharif.edu/ ־langari/

1 D. C. Tsui, H. L. Stormer, and A. C. Gossard, Phys. Rev. Lett. 48, 1559 (1982)

2 T. Hansson, V. Oganesyan, and S. Sondhi, Annals of Physics 313, 497 (2004)

3 X. Chen, Z.-C. Gu, and X.-G. Wen, Phys. Rev. B 82, 155138 (2010)

4 C. Nayak, S. H. Simon, A. Stern, M. Freedman, and S. Das Sarma, Rev. Mod. Phys. 80, 1083 (2008)

5 A. Kitaev, Annals of Physics 303, 2 (2003)

${ }^{6}$ X. G. Wen and Q. Niu, Phys. Rev. B 41, 9377 (1990)

7 X.-G. Wen, Quantum field theory of many-body systems: from the origin of sound to an origin of light and electrons (Oxford University Press, Oxford, 2007).

${ }^{8}$ H. Bombin and M. A. Martin-Delgado, Phys. Rev. Lett. 97, 180501 (2006)
${ }^{9} \mathrm{H}$. Bombin and M. A. Martin-Delgado, Phys. Rev. B 75, 075103 (2007)

$10 \mathrm{H}$. Bombin and M. A. Martin-Delgado, Phys. Rev. A 76, 012305 (2007)

11 M. Kargarian, Phys. Rev. A 78, 062312 (2008)

12 A. Kitaev and J. Preskill, Phys. Rev. Lett. 96, 110404 (2006)

13 M. Levin and X.-G. Wen, Phys. Rev. Lett. 96, 110405 (2006)

14 K. P. Schmidt, S. Dusuel, and J. Vidal, Phys. Rev. Lett. 100, 057208 (2008)

15 S. Dusuel, K. P. Schmidt, and J. Vidal, Phys. Rev. Lett. 100, 177204 (2008)

16 H. Bombin, M. Kargarian, and M. A. Martin-Delgado, Phys. Rev. B 80, 075111 (2009)

17 M. Kargarian, Phys. Rev. A 80, 012321 (2009)

18 M. Kamfor, S. Dusuel, J. Vidal, and K. P. Schmidt, Phys. Rev. B 89, 045411 (2014) 
19 K. P. Schmidt, Phys. Rev. B 88, 035118 (2013).

${ }^{20}$ S. Dusuel, K. P. Schmidt, J. Vidal, and R. L. Zaffino, Phys. Rev. B 78, 125102 (2008)

21 V. Karimipour, L. Memarzadeh, and P. Zarkeshian, Phys. Rev. A 87, 032322 (2013)

22 J. Vidal, S. Dusuel, and K. P. Schmidt, Phys. Rev. B 79, 033109 (2009)

23 J. Vidal, R. Thomale, K. P. Schmidt, and S. Dusuel, Phys. Rev. B 80, 081104 (2009)

24 S. S. Jahromi, M. Kargarian, S. F. Masoudi, and K. P. Schmidt, Phys. Rev. B 87, 094413 (2013).

25 S. S. Jahromi, S. F. Masoudi, M. Kargarian, and K. P. Schmidt, Phys. Rev. B 88, 214411 (2013).

26 F. Pollmann, A. M. Turner, E. Berg, and M. Oshikawa, Phys. Rev. B 81, 064439 (2010).

$27 \mathrm{X}$. Chen, Z.-C. Gu, and X.-G. Wen, Phys. Rev. B 83, 035107 (2011)

28 X. Chen, Z.-C. Gu, Phys. Rev. B 84, 235128 (2011)

29 Projective representation of a specific symmetry group, e.g., $G$ is accompanied by a phase factor. For instance, if elements $g, g^{\prime}$ and $g^{\prime \prime} \in G$ have the relation $g g^{\prime}=g^{\prime \prime}$, the corresponding projective representation becomes $U_{g} U_{g^{\prime}}=e^{i \theta} U_{g^{\prime \prime}}$.

${ }^{30}$ F. Pollmann and A. M. Turner, Phys. Rev. B 86, 125441 (2012).

${ }^{31}$ C.-Y. Huang, X. Chen, and F. Pollmann, arXiv:1312.3093 (2013)

32 M. P. Zaletel, arXiv:1309.7387 (2013)

33 L. Balents, Nature 464, 199 (2010)

${ }^{34}$ C. Arizmendi, A. Rizzo, L. Epele, and C. García Canal, Zeitschrift für Physik B Condensed Matter 83, 273 (1991)

35 P. Sen, S. Chakraborty, S. Dasgupta, and B. Chakrabarti, Zeitschrift für Physik B Condensed Matter 88, 333 (1992)

36 H. Rieger and G. Uimin, Zeitschrift für Physik B Condensed Matter

${ }^{37}$ R. Derian, A. Gendiar, and T. Nishino, Journal of the Physical Society of Japan 75, 114001 (2006)
${ }^{38}$ M. Beccaria, M. Campostrini, and A. Feo, Phys. Rev. B 73, 052402 (2006)

39 M. Beccaria, M. Campostrini, and A. Feo, Phys. Rev. B 76, 094410 (2007)

40 A. K. Chandra and S. Dasgupta, Phys. Rev. E 75, 021105 (2007)

41 A. K. Chandra and S. Dasgupta, Journal of Physics A: Mathematical and Theoretical 40, 6251 (2007)

42 A. Nagy, New Journal of Physics 13, 023015 (2011)

43 U. Schollwöck, Annals of Physics 326, 96 (2011).

44 G. Vidal, Phys. Rev. Lett. 98, 070201 (2007)

45 I. P. McCulloch, arXiv:0804.2509 (2008)

46 J. Villain, R. Bidaux, C. J.-P., and R. Conte, J. Phys. France 41, 1263 (1980)

47 E. F. Shender, Journal of Experimental and Theoretical Physics 56, 178 (1982)

48 R. Moessner, S. L. Sondhi, and P. Chandra, Phys. Rev. Lett. 84, 4457 (2000).

49 V. Karimipour, Phys. Rev. B 79, 214435 (2009)

${ }^{50}$ E. Lieb, T. Schultz, and D. Mattis, Annals of Physics 16, 407 (1961)

51 M. Karbach, G. Müller, and K. Wiele, Journal of Physics A: Mathematical and Theoretical 41, 205002 (2008).

52 J. A. Kjäll, M. P. Zaletel, R. S. K. Mong, J. H. Bardarson, and F. Pollmann, Phys. Rev. B 87, 235106 (2013)

53 P. Calabrese and J. Cardy, Journal of Statistical Mechanics: Theory and Experin

${ }^{54}$ H. W. J. Blöte and Y. Deng, Phys. Rev. E 66, 066110 (2002)

55 M. S. L. du Croo de Jongh and J. M. J. van Leeuwen, Phys. Rev. B 57, 8494 (1998)

56 M. Sadrzadeh and A. Langari, arXiv:1405.1233 (2014)

57 F. Verstraete and J. I. Cirac, Phys. Rev. B 73, 094423 (2006)

58 M. B. Hastings, Phys. Rev. Lett. 93, 140402 (2004)

${ }^{59}$ R. Orús and G. Vidal, Phys. Rev. B 78, 155117 (2008)

${ }^{60}$ H.-Q. Zhou, R. Orús, and G. Vidal,

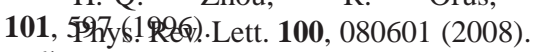

${ }^{61}$ M. Sanz, M. M. Wolf, D. Perez-Garcia, and J. I. Cirac, Phys. Rev. A 79, 042308 (2009) 